\title{
Dynamic instability and bifurcation of electrically actuated circular nanoplate considering surface behavior and small scale effect
}

\author{
W.D. Yang, F.P. Yang and X. Wang* \\ School of Naval Architecture, Ocean and Civil Engineering \\ (State Key Laboratory of Ocean Engineering) \\ Shanghai Jiao Tong University, Shanghai 200240, P. R. China
}

\begin{abstract}
In this paper, the dynamic pull-in instability and bifurcation characteristics of circular nanoplate subjected to electrostatic and Casimir forces are studied. Surface effect originates from high surface/volume ratio of nanostructures, where atoms at a free surface experience distinct local environments with respect to those in the bulk material. Thus, the surface free energy being negligible in classical elastic theory, becomes significant in dynamic behaviors of nanostructures. Based on Eringen's nonlocal elasticity and Gurtin-Murdoch surface model, the nonlinear governing equation of electrically actuated circular nanoplate is derived in polar coordinate. The closed-form solution of dynamic frequency and electrostatic voltage is obtained by utilizing the homotopy perturbation method (HPM). Furthermore, the coupling effects of nonlocal parameter and surface characteristics on the dynamic pull-in instability of circular nanoplate are investigated, and the nonlinear dynamics behaviors, time histories and phase diagrams of electrically actuated circular nanoplate are discussed. Some new results obtained in this work could be helpful in design of 2-D circular nanoplate-type actuator considering size-dependency and quantum vacuum fluctuation effects.
\end{abstract}

Keywords: Dynamic pull-in, Circular nanoplate, Bifurcation, Small scale effect, Surface behaviors, Homotopy perturbation method

\footnotetext{
* Corresponding author at: School of Naval Architecture, Ocean and Civil Engineering,

Shanghai Jiaotong University, Shanghai 200240, P. R. China.

Tel./fax: 086-021-54745367/086-021-54745367

E-mail address: xwang@sjtu.edu.cn (X. Wang).
} 


\section{Introduction}

The continuing miniaturization of electromechanical devices promotes the wide application of micro-/nano-electromechanical systems (MEMS/NEMS) in sensors [1, 2], actuators [3-7], resonators [8-10], switches [11-13], tweezers [14], oscillators [15, 16] and gyroscopes [17]. However, dynamic pull-in phenomenon as one critical electromechanical instability might lead actuated devices to easily lose stability and reliability, even result in adhesion, stiction, wear and failure [18]. Therefore, how to clearly understand the dynamic pull-in characteristics of actuated devices and effectively control the pull-in instability has become urgent in the design of MEMS/NEME-based devices.

In general, typical electrically actuated devices are composed of a parallel-plate capacitor, where the movable plate deforms towards to the substrate as electrostatic voltage increases. As the size of devices reduces to nanometer scale, dispersion forces such as van der Waals force and Casimir force become significantly important in electromechanical coupling behaviors of devices [19-25]. Both of van der Waals force and Casimir force originate from fluctuation of electromagnetic field, but based on different separation regimes. Furthermore, van der Waals force generally describes that a fluctuating dipole p1 induces a fluctuating electromagnetic dipole field, which in turn induces a fluctuating dipole $\mathrm{p} 2$ on a neighbor particle. The van der Waals force has two crucial approximations: (i) quasi-static approximation to ignore wave effect, and (ii) ignores multiple scattering if there are more than two particles [21, 26]. However, when the separation is much larger than the typical wavelength, the finite wave propagation speed of light must be taken into account. Also, the multiple scattering can be negligible for a sufficiently dilute gas or for weak polarizability, but it becomes very significant for interactions between two solid bodies. Therefore, the multiple scattering effects combined with wave retardation yield only the Casimir force [26], where the effect of van der Waals force may be ignored in studying the dynamic instability of electrostatically actuated nanoplate system with a pair of conducting parallel plates. 
Lin and Zhao [27] utilized a one degree of freedom mass-spring model to investigate the effect of Casimir force on static and dynamic pull-in instability of nano-actuators, and indicated that the movable beam would collapsed onto the substrate without any voltage at the two electrodes due to Casimir effect. Batra et al. [28] developed reduce-order models for electrically actuated circular and rectangular micro-plate with geometrical nonlinearity and Casimir force, where the vibrational behaviors of micro-plate subjected to Coulomb and the Casimir forces were analyzed and results showed that the first natural frequency equals zero at the pull-in voltage.

At the nanoscale, the dependence of material behaviors on size becomes a crucial factor in designing such nano-devices. The size-dependent properties of nanostructures have been confirmed in experimental observation and theoretical analysis[29, 30]. Classical elastic theory cannot capture the size-dependent characteristics of nanostructures; thus, some modified models with size-dependency are proposed. Among these, Eringen's nonlocal elastic theory[31, 32] has been widely employed in nanotechnology, in which the stress at a referenced point depends on the strains at all points in elastic body, [33-35]. Based on Eringen's nonlocal elastic theory, the size-dependent constitutive model depends on an internal characteristic length of materials (e.g., lattice parameter, granular distance), an external characteristic length (e.g., crack length, wave length), and their comparative ratios [36]. For macro structures, the internal characteristic length of materials and the ratio between internal and external characteristic lengths are too small, so that the effect of size dependency is negligible. Nevertheless, when the size of structure enters to nanometer scale and the ratio is comparative, the small scale parameters become significant. One key factor for utilizing the scale parameters in Eringen's nonlocal elastic theory to describe the scale effect of nanoscale materials is how to determine the correct scale parameter $e_{0} a$. Eringen [36] presented a method to determine the small scale parameter by comparing the results of plane waves from Born-Karman model of lattice dynamics and nonlocal elasticity theory. Zhang et al. [37] gave different scale parameters to solve the frequency of SWCNTs with different length-to-diameter ratios 
and chiral angels by comparing the flexural vibrations of single walled carbon nanotubes (SWCNTs) from Timoshenko beam model and MD simulation. By comparing the dynamic behaviors of bilayer graphene sheets (BLGSs) from MD simulations and nonlocal elastic models, Shen et al. [38] determined a scale parameter to describe the scale effect in solving the natural frequency of BLGSs.

In the MEMS/NEMS-based devices regime, Mousavi et al. [12] employed nonlocal Euler-Bernoulli beam theory to study the static pull-in instability of nano-switches under electrostatic and intermolecular forces, and showed that the nonlocal cantilever nano-beam displays stiffening effects whilst doubly-clamped nanobeam shows softening effect owing to the small scale effect. Arani et al. [39] considered the nonlocal beam model and geometric nonlinearity to investigate the static pull-in instability of boron nitride nano-switches (BNNSs) subjected to electrostatic and van der Waals forces. Wang et al. [40] used nonlocal plate model to study the small scale effect on the dynamic pull-in instability and frequency of rectangular graphene sheets under electrostatic and intermolecular forces.

Furthermore, the surface effect is one of the significant molecular effects at the atomic scale, showing size-dependency of nanostructures [41-44]. Because of distinct environment conditions, atoms locating at or near the free surface have different equilibrium requirements than those in the bulk of material. This difference gives rise to additional surface energy in the surface layer of material and surface residual stress. Gurtin and Murdoch[45, 46] developed an analytical model to incorporate the surface effects to characterize the mechanical response of nanostructures. Ansari et al. [47] used Gurtin-Murdoch model to analyze the static pull-in instability behaviors of hydrostatically and electrostatically actuated circular nanoplates considering surface behaviors. Yang et al. [48] incorporated nonlocal beam theory and surface effect to model carbon nanotubes-reinforced cantilever nano-actuator and analyzed the effect of Casimir force on static pull-in instability and free standing behaviors. In another paper, Yang et al. [49] studied the coupling impacts of surface stress, nonlocal parameter and thermal effect on pull-in of nano-switches. Recently, Sahmani and Bahrami[50] utilized a high-order shear deformation plate model to study the dynamic 
pull-in instability and free vibration properties of circular nanoplates including surface effect. They also tried to implement the nonlocal elasticity theory into the governing equation, but it is unfortunate that they cannot derive an explicit governing equation including surface stress and small scale effect, even the impact of Casimir force cannot be considered.

This work aims to employed nonlocal elasticity theory and surface effects to present a size-dependent analytical model of electrically actuated circular nanoplate and investigate the coupling effects of nonlocal parameter, surface elasticity modulus and surface residual stress on dynamic pull-in instability. The dynamic frequency and bifurcation characteristics of circular nanoplate subjected to electrostatic and Casimir forces are also studied. The nonlinear nonlocal governing equation is analytically solve by the homotopy perturbation method (HPM) [51] widely used to solve nonlinear physical problems [52-54]. Finally, to verify the accuracy of HPM, the comparison of results obtained by using the HPM, experiment and other numerical approaches is performed, and the results from different methods show a good agreement.

\section{Theory and model}

To capture the size-dependent characteristics of nanostructures, Eringen's nonlocal elasticity containing the long range force among atoms in body is utilized, in which the stress-strain relationship can be expressed as [55]

$$
\sigma_{i j}^{n l}(x)=\int H\left(\left|x-x^{\prime}\right|, e_{0} a / l\right) C_{i j k l} \varepsilon_{k l}\left(x^{\prime}\right) d V\left(x^{\prime}\right), \quad \forall x \in V
$$

where $\sigma_{i j}^{n l}, \varepsilon_{k l}$ and $C_{i j k l}$ denote the nonlocal stress, strain and fourth-order elasticity tensors, respectively. The term $H\left(\left|x-x^{\prime}\right|, e_{0} a / l\right)$ is the principal attenuation kernel function related to the nonlocal effects in terms of the Euclidean distance between the point $x$ and neighboring points $x^{\prime}$ within a domain $V .\left|x-x^{\prime}\right|$ is the distance between the two reference points $x$ and $x^{\prime} . e_{0} a / l$ represents nonlocal effects, $e_{0}$ denotes a material constant, $a$ is the internal characteristic length of material and $l$ 
is the external characteristic length of the material. The internal characteristic length represents the scale parameter such as length of C-C bond, lattice spacing or granular distance.

For the homogenous isotropic body without body forces, the nonlocal elasticity is reduced to following form as [55]

$$
\left[1-\left(e_{0} a\right)^{2} \nabla^{2}\right] \sigma^{n l}=C: \varepsilon
$$

where $\left(e_{0} a\right)$ represents the nonlocal parameter, $\nabla^{2}$ represents the Laplace differential operator. For axisymmetric deformation of circular nanoplate, the size-dependent constitutive equation based on nonlocal elasticity is expressed in polar coordinates, as follows:

$$
\begin{aligned}
& \sigma_{r}^{n l}-\left(e_{0} a\right)^{2} \nabla^{2} \sigma_{r}^{n l}=\frac{E}{1-v^{2}}\left(\varepsilon_{r}+v \varepsilon_{\theta}\right) \\
& \sigma_{\theta}^{n l}-\left(e_{0} a\right)^{2} \nabla^{2} \sigma_{\theta}^{n l}=\frac{E}{1-v^{2}}\left(v \varepsilon_{r}+\varepsilon_{\theta}\right)
\end{aligned}
$$

where $r$ is the radial variable, $\sigma_{r}^{n l}$ and $\sigma_{\theta}^{n l}$ denote the nonlocal radial and circumferential counterparts of stress tensor, $\varepsilon_{r}$ and $\varepsilon_{\theta}$ are radial and circumferential strains, and $v$ represents the Poisson's ratio, respectively. The corresponding displacements of circular nanoplate are described as

$$
u_{z}(r, t)=w(r, t), u_{r}(r, t)=-z \frac{\partial u_{z}}{\partial r}, u_{\theta}(r, t)=0
$$

where $u_{z}, u_{r}$ and $u_{\theta}$ are the displacements in the transverse z-direction, radial $r$ direction and circumferential direction, respectively. Based on the Kirchhoff plate theory, the radial and circumferential strains of axisymmetric circular nanoplate are expressed as

$$
\varepsilon_{r}=-z \frac{\partial^{2} w(r, t)}{\partial r^{2}}, \varepsilon_{\theta}=-\frac{z}{r} \frac{\partial w(r, t)}{\partial r}, \varepsilon_{r \theta}=0
$$

Moreover, the circular nanoplate is considered to have an elastic surface layer with zero thickness, and the surface layer has specific material characteristics incorporating surface energy effects and is assumed to be perfectly bonded to the bulk material, as shown in Fig. 1. According to Gurtin-Murdoch model [47], the constitutive relations 
of surface layer are expressed as

$$
\sigma_{\alpha \beta}^{s \pm}=\tau^{s \pm} \delta_{\alpha \beta}+\lambda^{s \pm} \varepsilon_{\alpha \beta}^{s \pm} \delta_{\alpha \beta}+2 \mu^{s \pm} \varepsilon_{\alpha \beta}^{s \pm}, \quad \sigma_{\gamma z}^{s \pm}=\tau^{s \pm} \frac{\partial w}{\partial \gamma}
$$

where $\sigma_{\alpha \beta}^{s \pm}, \varepsilon_{\alpha \beta}^{s \pm}, \tau^{s \pm}$ denote the stress, strain, residual tension of surface layers; $\lambda^{s \pm}, \mu^{s \pm}$ are the surface Lame constants; $\delta_{\alpha \beta}$ is the Kronecker delta. In the above equations, the Greek subscripts represent the values of $r$ and $\theta$. From Eqs. (5) and (6), the surface stresses can be expressed as

$$
\begin{gathered}
\sigma_{r}^{s \pm}=\tau^{s} \mp \frac{E^{s} h}{2\left(1-v^{2}\right)}\left(\frac{\partial^{2} w}{\partial r^{2}}+\frac{v}{r} \frac{\partial w}{\partial r}\right) \\
\sigma_{\theta}^{s \pm}=\tau^{s} \mp \frac{E^{s} h}{2\left(1-v^{2}\right)}\left(v \frac{\partial^{2} w}{\partial r^{2}}+\frac{1}{r} \frac{\partial w}{\partial r}\right) \\
\sigma_{r z}^{s \pm}=\tau^{s} \frac{\partial w}{\partial r}
\end{gathered}
$$

where $E^{s}$ represents the surface modulus and $h$ is the thickness of circular nanoplate. Using the following stress resultants for the derivation of nonlocal circular nanoplate considering surface effects, leads to

$$
\begin{aligned}
& M_{r}=\int_{-h / 2}^{h / 2} \sigma_{r}^{n l} z d z+\frac{h}{2}\left(\sigma_{r}^{s+}-\sigma_{r}^{s-}\right) \\
& M_{\theta}=\int_{-h / 2}^{h / 2} \sigma_{\theta}^{n l} z d z+\frac{h}{2}\left(\sigma_{\theta}^{s+}-\sigma_{\theta}^{s-}\right)
\end{aligned}
$$

where $M_{r}$ and $M_{\theta}$ denote radial and circumferential bending moments, respectively. Substituting Eqs.(7a c) into Eqs.(8a,b) in conjunction with Eqs.(3a,b), the nonlocal stress resultants is given by

$$
\begin{aligned}
& M_{r}-\left(e_{0} a\right)^{2} \nabla^{2} M_{r}=-D^{*}\left(\frac{\partial^{2} w}{\partial r^{2}}+\frac{v}{r} \frac{\partial w}{\partial r}\right) \\
& M_{\theta}-\left(e_{0} a\right)^{2} \nabla^{2} M_{\theta}=-D^{*}\left(v \frac{\partial^{2} w}{\partial r^{2}}+\frac{1}{r} \frac{\partial w}{\partial r}\right)
\end{aligned}
$$

where

$$
D^{*}=k^{s} D, k^{s}=1+\frac{6 E^{s}}{E h}, \text { and } D=\frac{E h^{3}}{12\left(1-v^{2}\right)}
$$


In the above formula, $k^{s}$ represents the ratio of surface rigidity versus bulk and $D$ is the flexural rigidity of circular nanoplate. Note that the stress resultants in Eqs. (9a, b) are reduced to the classical circular plate model when the surface elastic modulus $E^{s}$ and nonlocal parameters are set as zero. It is seen that when bulk thickness decreases into the nanoscale, the ratio $k^{s}$ becomes significant, so that the surface elasticity has a hardening effect on flexural rigidity of nanoplate. The equilibrium equations of axisymmetric circular nanoplate is expressed as [56]

$$
\begin{gathered}
\frac{\partial M_{r}}{\partial r}+\frac{M_{r}-M_{\theta}}{r}+\sigma_{r z}^{s+}-\sigma_{r z}^{s-}=Q_{r} \\
\frac{1}{r} \frac{\partial}{\partial r}\left(r Q_{r}\right)+\frac{N_{r}}{r} \frac{\partial}{\partial r}\left(r \frac{\partial w}{\partial r}\right)+F_{d i s}=\rho h \frac{\partial^{2} w}{\partial t^{2}}
\end{gathered}
$$

where $F_{d i s}$ is the sum of transverse distributed loads; $\rho$ is the mass density of circular nanoplate. For circular nanoplate under uniform radial mechanical loading coupling with environment temperature change, the radial in-plane force $N_{r}$.is expressed as

$$
N_{r}=N_{m e}+N_{T}
$$

where $N_{m e}$ and $N_{T}$ are the radial forces induced by the mechanical loading and the thermal effect from the environment temperature change, respectively. According to the thermal elasticity theory[57], the thermal radial force is given by

$$
N_{T}=-\left(\frac{E \alpha h}{1-v}\right) \Delta T
$$

where $\alpha$ and $\Delta T$ are the coefficient of thermal expansion (CTE) and temperature change. Decoupling Eqs.(9a,b) and Eqs.(11a,b) leads to the nonlocal dynamic governing equation of circular nanoplate considering surface elasticity and surface residual stress[58], as follows: 


$$
\begin{aligned}
D^{*} & \left(\frac{\partial^{4} w}{\partial r^{4}}+\frac{2}{r} \frac{\partial^{3} w}{\partial r^{3}}-\frac{1}{r^{2}} \frac{\partial^{2} w}{\partial r^{2}}+\frac{1}{r^{3}} \frac{\partial w}{\partial r}\right)-\left(e_{0} a\right)^{2}\left[D^{*}+\left(e a_{0}\right)^{2}\left(N_{r}+2 \tau^{s}\right)\right] \frac{\partial^{6} w}{\partial r^{6}} \\
& -\left(N_{r}+2 \tau^{s}\right)\left(\frac{\partial^{2} w}{\partial r^{2}}+\frac{1}{r} \frac{\partial w}{\partial r}\right)-\frac{7}{r}\left(e_{0} a\right)^{2}\left[D^{*}+\left(e_{0} a\right)^{2}\left(N_{r}+2 \tau^{s}\right)\right] \frac{\partial^{5} w}{\partial r^{5}} \\
& +\left(e_{0} a\right)^{2}\left\{2\left(N_{r}+2 \tau^{s}\right)-\frac{1}{r^{2}}\left[D^{*}(3+2 v)+5\left(e_{0} a\right)^{2}\left(N_{r}+2 \tau^{s}\right)\right]\right\} \frac{\partial^{4} w}{\partial r^{4}} \\
+ & \frac{2}{r}\left(e_{0} a\right)^{2}\left\{4\left(N_{r}+2 \tau^{s}\right)+\frac{1}{r^{2}}\left[D^{*}(1+2 v)+3\left(e_{0} a\right)^{2}\left(N_{r}+2 \tau^{s}\right)\right]\right\} \frac{\partial^{3} w}{\partial r^{3}} \\
+ & \frac{1}{r^{2}}\left(e_{0} a\right)^{2}\left\{2\left(N_{r}+2 \tau^{s}\right)+\frac{3}{r^{2}}\left[D^{*}(1-2 v)-\left(e_{0} a\right)^{2}\left(N_{r}+2 \tau^{s}\right)\right]\right\} \frac{\partial^{2} w}{\partial r^{2}} \\
& -\frac{1}{r^{3}}\left(e_{0} a\right)^{2}\left\{2\left(N_{r}+2 \tau^{s}\right)+\frac{3}{r^{2}}\left[D^{*}(1-2 v)-\left(e_{0} a\right)^{2}\left(N_{r}+2 \tau^{s}\right)\right]\right\} \frac{\partial w}{\partial r} \\
+ & m h\left[\left(e_{0} a\right)^{4} \frac{\partial^{6} w}{\partial r^{4} \partial t^{2}}+\left(e_{0} a\right)^{4} \frac{6}{r} \frac{\partial^{5} w}{\partial r^{3} \partial t^{2}}-\left(e_{0} a\right)^{2} \frac{1}{r^{2}}\left(2 r^{2}-3 \mu\right) \frac{\partial^{4} w}{\partial r^{2} \partial t^{2}}\right. \\
& \left.-\left(e_{0} a\right)^{2} \frac{3}{r^{3}}\left(2 r^{2}+\left(e_{0} a\right)^{2}\right) \frac{\partial^{3} w}{\partial r \partial t^{2}}+\frac{\partial^{2} w}{\partial t^{2}}\right]-\left(e_{0} a\right)^{4} \frac{\partial^{4} F_{d i s}}{\partial r^{4}}-\left(e_{0} a\right)^{4} \frac{6}{r} \frac{\partial^{3} F_{d i s}}{\partial r^{3}} \\
& -\left(e_{0} a\right)^{2} \frac{1}{r^{2}}\left[2 r^{2}-3\left(e_{0} a\right)^{2}\right] \frac{\partial^{2} F_{d i s}}{\partial r^{2}}+\left(e_{0} a\right)^{2} \frac{3}{r^{3}}\left[2 r^{2}+\left(e_{0} a\right)^{2}\right] \frac{\partial F_{d i s}}{\partial r}-F_{d i s}=0
\end{aligned}
$$

For the electrically actuated circular nanoplate, the gap between the electrodes is small compared to nanoplate radius, thus the fringing field effect can be ignored and the distributed electrostatic force can be written as [27]

$$
F_{e}=\frac{\varepsilon_{0} \bar{V}_{e}^{2}}{2\left[g_{0}-w(r)\right]^{2}}
$$

where $\varepsilon_{0}=8.854 \times 10^{-12} C^{2} N^{-1} m^{-2}$ is the permittivity of vacuum, $\bar{V}_{e}$ is the applied electrostatic voltage, and $g_{0}$ is the initial gap between circular nanoplate and substrate.

Moreover, Casimir force as the macro effect of quantum field fluctuation is defined as [48]

$$
F_{C}=\frac{\pi^{2} h c_{0}}{240\left[g_{0}-w(r)\right]^{4}}
$$

where $\mathrm{h}=1.055 \times 10^{-34} \mathrm{JS}$ is the Plank's constant divided by $2 \pi$ and 
$c=3 \times 10^{8} \mathrm{~m} \cdot \mathrm{s}^{-1}$ is the speed of light. The transverse distributed load $F_{\text {dis }}$ in Eq. (14) is the sum of electrical actuation force and Casimir force exerted on the circular nanoplate, which is written as $F_{d i s}=F_{e}+F_{C}$.

For further parametrical investigation, non-dimensional variables are given by

$$
\begin{gathered}
W=\frac{w}{g_{0}}, \quad \rho=\frac{r}{R}, \quad \mu=\frac{e_{0} a}{R}, \quad N_{0}=\frac{N_{r} R^{2}}{D}, \tau_{0}^{s}=\frac{\tau^{s} R^{2}}{D}, \\
\chi=\frac{t}{R^{2}} \sqrt{\frac{D}{\rho h}}, \quad K_{2}=\frac{\varepsilon_{0} \bar{V}_{e}^{2} R^{4}}{2 g_{0}^{3} D}, \quad K_{4}=\frac{\pi^{2} \hbar c_{0} R^{4}}{240 g_{0}^{5} D}
\end{gathered}
$$

Substituting Eq. (17) into Eq. (14) leads to the non-dimensional nonlocal dynamic governing equation of circular nanoplate, as follows

$$
\begin{aligned}
k^{s}( & \left.\frac{\partial^{4} W}{\partial \rho^{4}}+\frac{2}{\rho} \frac{\partial^{3} W}{\partial \rho^{3}}-\frac{1}{\rho^{2}} \frac{\partial^{2} W}{\partial \rho^{2}}+\frac{1}{\rho^{3}} \frac{\partial W}{\partial \rho}\right)-\left(N_{0}+2 \tau_{0}^{s}\right)\left(\frac{\partial^{2} W}{\partial \rho^{2}}+\frac{1}{\rho} \frac{\partial W}{\partial \rho}\right) \\
- & \mu^{2}\left[k^{s}+\mu^{2}\left(N_{0}+2 \tau_{0}^{s}\right)\right] \frac{\partial^{6} W}{\partial \rho^{6}}-\frac{7 \mu^{2}}{\rho}\left[k^{s}+\mu^{2}\left(N_{0}+2 \tau_{0}^{s}\right)\right] \frac{\partial^{5} W}{\partial \rho^{5}} \\
+ & \mu^{2}\left\{2\left(N_{0}+2 \tau_{0}^{s}\right)-\frac{1}{\rho^{2}}\left[k^{s}(3+2 v)+5 \mu^{2}\left(N_{0}+2 \tau_{0}^{s}\right)\right]\right\} \frac{\partial^{4} W}{\partial \rho^{4}} \\
+ & \frac{2 \mu^{2}}{\rho}\left\{4\left(N_{0}+2 \tau_{0}^{s}\right)+\frac{1}{\rho^{2}}\left[k^{s}(1+2 v)+3 \mu^{2}\left(N_{0}+2 \tau_{0}^{s}\right)\right]\right\} \frac{\partial^{3} W}{\partial \rho^{3}} \\
+ & \frac{\mu^{2}}{\rho^{2}}\left\{2\left(N_{0}+2 \tau_{0}^{s}\right)+\frac{3}{\rho^{2}}\left[k^{s}(1-2 v)-\mu^{2}\left(N_{0}+2 \tau_{0}^{s}\right)\right]\right\} \frac{\partial^{2} W}{\partial \rho^{2}} \\
- & \frac{\mu^{2}}{\rho^{3}}\left\{2\left(N_{0}+2 \tau_{0}^{s}\right)+\frac{3}{\rho^{2}}\left[k^{s}(1-2 v)-\mu^{2}\left(N_{0}+2 \tau_{0}^{s}\right)\right]\right\} \frac{\partial W}{\partial \rho} \\
+ & \mu^{4} \frac{\partial^{6} W}{\partial \rho^{4} \partial \chi^{2}}+\frac{6 \mu^{4}}{\rho} \frac{\partial^{5} W}{\partial \rho^{3} \partial \chi^{2}}-\frac{\mu^{2}}{\rho^{2}}\left(2 \rho^{2}-3 \mu^{2}\right) \frac{\partial^{4} W}{\partial \rho^{2} \partial \chi^{2}} \\
- & \frac{3 \mu^{2}}{\rho^{3}}\left(2 \rho^{2}+\mu^{2}\right) \frac{\partial^{3} W}{\partial \rho \partial \chi^{2}}+\frac{\partial^{2} W}{\partial \chi^{2}}-\mu^{4} \frac{\partial^{4} \hat{F}_{d i s}}{\partial \rho^{4}}-\frac{6 \mu^{4}}{\rho} \frac{\partial^{3} \hat{F}_{d i s}}{\partial \rho^{3}} \\
- & \frac{\mu^{2}}{\rho^{2}}\left(2 \rho^{2}-3 \mu^{2}\right) \frac{\partial^{2} \hat{F}_{d i s}}{\partial \rho^{2}}+\frac{3 \mu^{2}}{\rho^{3}}\left(2 \rho^{2}+\mu^{2}\right) \frac{\partial \hat{F}_{d i s}}{\partial \rho}-\hat{F}_{d i s}=0
\end{aligned}
$$

where $\hat{F}_{\text {dis }}$ denotes the sum of non-dimensional transverse distributed forces.

\section{Solution procedure}




\subsection{Nonlinear dynamic equations}

Utilizing Eigen function expansion, the solution of Eq. (18) is considered as

$$
W(\rho, T)=\sum_{m=1}^{N} \eta_{m}(\chi) \phi_{m}(\rho)
$$

where $\phi_{m}(\rho)$ is the $m$-th mode shape function, $\eta_{m}(\chi)$ is the $m$-th coefficient function for the $m$-th shape function, and $N$ is the number of chosen shape functions. As $N$ approaches a convergence value, the approximation in Eq.(19) becomes exact. For the axisymmetric circular plate satisfying the clamped boundary conditions, the linear undamped modes of shape functions $\phi_{m}(\rho)$ is expressed as[59]

$$
\phi_{m}(\rho)=\left(\frac{J_{0}\left(\rho \sqrt{\omega_{m}}\right)}{J_{0}\left(\sqrt{\omega_{m}}\right)}-\frac{I_{0}\left(\rho \sqrt{\omega_{m}}\right)}{I_{0}\left(\sqrt{\omega_{m}}\right)}\right)
$$

where $J_{0}$ is the Bessel function of the first kind, $I_{0}$ is the modified Bessel function of the first kind, and $\omega_{m}$ is the known $m$-th frequency. The mode shapes are orthonormal, satisfying

$$
\int_{0}^{1} \rho \phi_{m}(\rho) \phi_{n}(\rho) d \rho=\delta_{m n}
$$

where $\delta_{m n}$ represents the Kronecker delta. The fourth-order Taylor's series expansion of distributed forces is proved to have excellent accuracy to analytically study dynamic pull-in instability of nano-devices [60]. Substituting Eq. (19) into Eq. (18), multiplying updated Eq. (18) with $\rho^{4}$ to avoid the singularity of integration, then multiplying every term by $\rho \phi_{n}(\rho)$ over $\rho \in[0,1]$, and using the orthogonality condition of Eq. (21), lead to the governing equation of electrically actuated circular nanoplate as

$$
\begin{aligned}
& \sum_{k=1}^{N} \Psi_{0} \ddot{\eta}_{k}(\chi)+\Psi_{1}+\sum_{k=1}^{N} \Psi_{2} \eta_{k}(\chi)+\sum_{k=1}^{N} \sum_{m=1}^{N} \Psi_{3} \eta_{k}(\chi) \eta_{m}(\chi)+\sum_{k=1}^{N} \sum_{m=1}^{N} \sum_{l=1}^{N} \Psi_{4} \eta_{k}(\chi) \eta_{m}(\chi) \eta_{l}(\chi) \\
& +\sum_{k=1}^{N} \sum_{m=1}^{N} \sum_{l=1}^{N} \sum_{n=1}^{N} \Psi_{5} \eta_{k}(\chi) \eta_{m}(\chi) \eta_{l}(\chi) \eta_{n}(\chi)=0
\end{aligned}
$$


where $\Psi_{k}, k \in[0,5]$ are given in Appendix A.

\subsection{Application of homotopy perturbation method}

It is well known that well-established numerical techniques in literatures can be easily applied to solve the problems about dynamic instability of nanostructures. However, it is difficult to get generalizations of the problem from numerical results due to the results obtained from the numerical techniques are limited to fixed calculation parameters. In order to clearly describe the roles of some parameters of interest in a physical process, the homotopy perturbation method can be utilized to serves this purpose well in this paper, which is an effectively approximate method. The roles of some important controlled parameters for the dynamic instability and bifurcation of electrically actuated circular nanoplate, such as small scale parameter, surface effect, applied voltage, thermal environment, etc., can clearly be revealed from the present solution.

For Eq. (22), considering the dynamic behaviors of circular nanoplate mainly depend on the first term of eigen function expansion gives

$$
\ddot{\eta}_{1}(\chi)+\psi_{1}+\psi_{2} \eta_{1}(\chi)+\psi_{3}\left[\eta_{1}(\chi)\right]^{2}+\psi_{4}\left[\eta_{1}(\chi)\right]^{3}+\psi_{5}\left[\eta_{1}(\chi)\right]^{4}=0
$$

where $\psi_{k}=\Psi_{k} / \Psi_{0}, k \in[1,5]$. Based on the basic idea of HPM (homotopy method) [51], the homotopy equation can be constructed to solve Eq. (23), as follows:

$$
\mathscr{H}\left(\eta_{1}, p\right)=(1-p)\left[\ddot{\eta}_{1}+\psi_{1} \eta_{1}\right]+p\left[\ddot{\eta}_{1}+\psi_{0}+\psi_{1} \eta_{1}+\psi_{2} \eta_{1}^{2}+\psi_{3} \eta_{1}^{3}+\psi_{4} \eta_{1}^{4}\right]=0
$$

Assume the solution of Eq.(23) has the following form as

$$
\eta_{1}(\chi)=\eta_{1,0}(\chi)+p \eta_{1,1}(\chi)+p^{2} \eta_{1,2}(\chi)+\mathrm{L}
$$

where the coefficient of $\eta_{1}$ is expanded into a series of $p$, as follows

$$
\psi_{1}=\omega^{2}-p \omega_{1}-p^{2} \omega_{2}-\mathrm{L}
$$

Substituting Eq. (25) and Eq. (26) into Eq. (24), and equating the terms with the identical powers of $p$, 


$$
\begin{aligned}
p^{0}: & (\chi)+\omega^{2} \eta_{1,0}(\chi)=0, \quad \eta_{1,0}(0)=A, \quad \&_{1,0}(0)=0, \\
p^{1}: & (\chi)+\omega^{2} \eta_{1,1}(\chi)=\omega_{1} \eta_{1,0}(\chi)-\left[\psi_{0}+\psi_{2}\left(\eta_{1,0}(\chi)\right)^{2}+\psi_{3}\left(\eta_{1,0}(\chi)\right)^{3}+\psi_{4}\left(\eta_{1,0}(\chi)\right)^{4}\right], \\
& \eta_{1,1}(0)=0, \quad(27 \mathrm{a}) \\
p^{2}: & (\chi)+\omega^{2} \eta_{1,2}(\chi)=0, \\
& \left.+4 \psi_{4}\left(\eta_{1,0}(\chi)\right)^{3} \eta_{1,1}(\chi)\right], \quad \eta_{1,2}(0)=0, \quad \eta_{1,2}(0)=0
\end{aligned}
$$

Firstly, the solution of Eq. (27a) is written as

$$
\eta_{1,0}(\chi)=A \cos (\omega \chi)
$$

Because the solution of Eq. (27b) should not incorporate the secular term $\cos (\omega \chi)$, substituting Eq.(28) into Eq. (27b) yields

$$
\begin{gathered}
(\chi)+\omega^{2} \eta_{1,1}(\chi)=\left(\omega_{1} A-\frac{3}{4} \psi_{3} A^{3}\right) \cos (\omega \chi)-\left(\frac{1}{2} \psi_{2} A^{2}+\frac{1}{2} \psi_{4} A^{4}\right) \cos (2 \omega \chi) \\
-\frac{1}{2} \psi_{2} A^{2}-\frac{3}{8} \psi_{4} A^{4}-\psi_{0}-\frac{1}{4} \psi_{3} A^{3} \cos (3 \omega \chi)-\frac{1}{8} \psi_{4} A^{4} \cos (4 \omega \chi)
\end{gathered}
$$

Letting the secular terms of Eq. (29) be zero, leading to

$$
\omega_{1}=\frac{3}{4} \psi_{3} A^{2}
$$

Then, solving the updated Eq. (29) gives one second-order approximated solution of $\eta_{1,1}(\chi)$ as

$$
\begin{aligned}
\eta_{1,1}(\chi)= & \frac{1}{480 \omega^{2}}\left[\left(96 \psi_{4} A^{4}+160 \psi_{2} A^{2}-15 \psi_{3} A^{3}+480 \psi_{0}\right) \cos (\omega \chi)\right. \\
& +\left(80 \psi_{4} A^{4}+80 \psi_{2} A^{2}\right) \cos (2 \omega \chi)+15 \psi_{3} A^{3} \cos (3 \omega \chi) \\
& \left.+4 \psi_{4} A^{4} \cos (4 \omega \chi)-\left(480 \psi_{0}+180 \psi_{4} A^{4}+240 \psi_{2} A^{2}\right)\right]
\end{aligned}
$$

In addition, Eq. (26) with two terms approximation of power series when $p \rightarrow 1$,

$$
\omega_{2}=\omega^{2}-\psi_{1}-\omega_{1}
$$

Substituting Eq. (32) into Eq. (27c) for $\eta_{1,2}(\chi)$ and eliminating the secular terms, the frequency-amplitude equation is expressed as 


$$
\begin{gathered}
\omega^{4} A-\psi_{1} \omega^{2} A-\frac{3}{4} \psi_{3} \omega^{2} A^{3}+2 \psi_{0} \psi_{2} A+\frac{5}{6} \psi_{2}^{2} A^{3}-\frac{3}{2} \psi_{0} \psi_{3} A^{2}-\frac{1}{2} \psi_{2} \psi_{3} A^{4} \\
+\frac{3}{128} \psi_{3}^{2} A^{5}+3 \psi_{0} \psi_{4} A^{3}+\frac{7}{4} \psi_{2} \psi_{4} A^{5}-\frac{3}{10} \psi_{3} \psi_{4} A^{6}+\frac{63}{80} \psi_{4}^{2} A^{7}=0
\end{gathered}
$$

Finally, by solving Eq. (33) respect to the fundamental frequency $\omega$, the second-order frequency-amplitude relationship function can be easily determined.

\subsection{Bifurcation and dynamic stability}

To analyze the bifurcation characteristics of autonomous system, letting $\dot{\eta}_{1}(\chi)=\dot{q}_{1}(\chi)=q_{2}$ and substituting them into Eq. (23) lead to

$$
\left\{\begin{array}{c}
\dot{q}_{1}=q_{2} \\
\dot{q}_{2}=-f\left(q_{1}, q_{2}\right)
\end{array}\right.
$$

where $f\left(q_{1}, q_{2}\right)=\psi_{1}+\psi_{2} q_{1}+\psi_{3} q_{1}^{2}+\psi_{4} q_{1}^{3}+\psi_{5} q_{1}^{4}$. At the equilibrium points, the dynamic system is at rest, and the equilibrium points are computed by $q_{2}=0$ or $f\left(q_{1}, q_{2}\right)=0$. To check the stability of equilibrium points, the corresponding Jacobian matrix is expressed as

$$
J=\left[\begin{array}{cc}
0 & 1 \\
\psi_{2}+2 \psi_{3} q_{1}+3 \psi_{4} q_{1}^{2}+4 \psi_{5} q_{1}^{3} & 0
\end{array}\right]
$$

The corresponding eigenvalue of Jacobian matrix at $q_{1}=q_{1,1}$ satisfies $\lambda^{2}-\psi_{2}-2 \psi_{3} q_{1}-3 \psi_{4} q_{1}^{2}-4 \psi_{5} q_{1}^{3}=0$. For $\lambda^{2}<0$, the system has two pure imaginary roots and has the equilibrium point $\left(q_{1,1}, K_{2}\right)$ as the center point. For the other equilibrium points $\left(q_{1,2}, K_{2}\right)$, its eigenvalue satisfies $\lambda^{2}>0$ and the system has two real eigenvalues, where one is positive and the other one is negative. This means that this equilibrium point is an unstable saddle point.

Note that the fixed points or physically equilibrium positions of governing equation (23) exist in $\eta_{1} \in[0,1]$, but some points as solution of the governing equation (23) might exist outside the domain $[0,1]$. Actually, the number and positions fixed points 
and phase diagrams of dynamic system can be obtained by numerical analysis for the following equation:

$$
f\left(q_{1}, q_{2}\right)=\psi_{1}+\psi_{2} q_{1}+\psi_{3} q_{1}^{2}+\psi_{4} q_{1}^{3}+\psi_{5} q_{1}^{4}=0
$$

The positions of fixed points depending on $\left\{\psi_{m}\right\}, m \in[1,5]$ are considered as a function of applied electrostatic voltage, dispersion forces and physical parameters of electrically actuated circular nanoplate. When dispersion forces and physical parameters are given, the dynamic stability of circular nanoplate is dependent on electrostatic voltage.

\section{Results and discussion}

\subsection{Validation of HPM with numerical method}

In order to investigate the convergence and accuracy of HPM used to solve the dynamic pull-in instability considering the influence of Casimir force, the comparison of results from HPM and Runge-Kutta numerical method is performed. Table 1 lists the deflection of circular nanoplate with the influence of Casimir force $\left(\alpha_{4}=1\right)$ under different initial amplitudes and electrostatic voltages when the non-dimensional time is taken as $\chi=0.1$. It is observed that the deflections of circular nanoplate computed by second-order HPM well agree with those computed by Runge-Kutta numerical method. Even if the errors of results between HPM and Runge-Kutta numerical method slightly increase with the increment of initial amplitude, the largest error of deflection at initial amplitude $A=0.20$ and electrostatic voltage $K_{2}=5.0$ is only $3.79 \%$.

To further prove that the HPM in this paper is effective employed to investigate the dynamic pull-in of circular plate, the dynamic pull-in voltages of micro-plate obtained by the present HPM is compared to those from other methods in Refs.[50-52]. Table 2 gives the geometrical and material parameters of micro-plate for validation. The comparisons of the dynamic pull-in voltage obtained by HPM with those of experiment [61], finite element method (FEM) simulation [62], and step-by-step 
linearization method (SSLM) with 20 nodes[63] are list in Table 3. The results show that the result from HPM has good agreement with those from experiment and other numerical methods in literatures.

\subsection{Dynamic frequency and pull-in voltage}

From the nonlinear dynamic equation (34), the relation of dynamic frequency and initial amplitude of circular nanoplate can be easily determined. This section focused on the coupling influences of various physical parameters on the dynamic pull-in voltage of circular nanoplate. The relevant material and geometrical parameters of circular nanoplate in examples calculation are given in Table 4.

Fig. 2 illustrates the influences of the surface elasticity on the dynamic pull-in voltage and non-dimensional fundamental frequency of circular nanoplate. Due to the lack of existing experimental data, four different values of the surface elasticity in the range of $0-10 \mathrm{nN} / \mathrm{nm}$ are considered to investigate the pull-in voltage. It is observed that the frequency gradually decreases as the applied voltage increases; when the voltage continue to increase, the frequency would suddenly drop to zero, namely, the pull-in instability occurs. Furthermore, under the identical voltage, the frequency increases as the value of surface elasticity increases. The surface elasticity enhances the structural stiffness of circular nanoplate, thus the pull-in voltage with large surface elasticity is higher than that with small surface elasticity.

Fig. 3 shows the effect of surface residual stress on the pull-in voltage and non-dimensional frequency of circular nanoplate. The values of surface residual stress are considered in the range $0-1.0 \mathrm{nN} / \mathrm{nm}$. As the surface residual stress increases, the pull-in voltage of circular nanoplate reduces. The surface residual stress also decreases the fundamental frequency of circular nanoplate under the same applied voltage.

Fig. 4 and Fig. 5 depict that influences of the radial mechanical loading and environment temperature change on the dynamic pull-in voltage and fundamental frequency, respectively. From Fig. 4, the negative values of radial loading represent tensile stress while the positive values of radial loading are compressive stress. This 
means the radial compressive loading reduces the dynamic pull-in voltage and frequency of circular nanoplate. It is seen from Fig. 5 that as the temperature change increases, both of the pull-in voltage and frequency reduce, which means the increase of temperature change shows the compressive stress in radial direction of circular nanoplate.

Fig. 6 illustrates the effect of nonlocal parameter on the dynamic pull-in voltage and frequency of circular nanoplate. According to the MD simulation results, the values of nonlocal parameter $\left(e_{0} a\right)^{2}$ are in the range $0-3 \mathrm{~nm}^{2}$. It is observed that the pull-in voltage reduces with the increment of nonlocal parameter, and under the same voltage, the frequency declines as the nonlocal parameter increase. This means that the nonlocal parameter induces the decrease of frequency and pull-in voltage of circular nanoplate.

To study the coupling influence of surface elasticity and surface residual stress in conjunction with the nonlocal parameter on the dynamic behaviors of circular nanoplate, the non-dimensional fundamental frequency versus nonlocal parameters for different surface elasticity and surface residual stress is plotted in Fig. 7. It is seen that the frequency declines as the nonlocal parameter for all selection of surface elasticity and residual stress, which means the effect of nonlocal parameter is significant on the frequency of circular nanoplate. For the identical nonlocal parameter, the surface elasticity enhances the structural stiffness whereas the increment of surface residual stress reduces the frequency of circular nanoplate.

Fig. 8 depicts the frequency versus nonlocal parameter for different radial mechanical loadings and temperature changes. Herein, the positive temperature change and radial compressive loading are considered. The results show that the frequency of circular nanoplate with the clamped boundary decreases as the temperature changes and mechanical compression loading increase.

The influences of ratio of radius-to-thickness on non-dimensional frequency and voltage of electrostatically actuated circular nanoplate are studied, shown as in Fig. 9. The non-dimensional frequency decreases with the increment of ratio of 
radius-to-thickness at the identical applied voltage. Moreover, the effects of nonlocal parameter on non-dimensional frequency for different ratios of radius-to-thickness are investigated in Fig. 10. As the nonlocal parameters and ratio of radius-to-thickness increase, the non-dimensional frequency declines. Based on Eringen's nonlocal elasticity, the nonlocal parameters are determined by the ratios of internal characteristic length to external characteristic length, and thus the nonlocal parameter $\mu=e_{0} a / R$ decreases when radius of circular nanoplate gradually increase. Therefore, the influence of nonlocal parameters become weakening as the size of device system increases.

\subsection{Dynamic instability and bifurcation}

The coupling effects of the applied voltage and Casimir force on the transition history of equilibrium points are summarized in phase diagrams of circular nanoplate for different initial conditions. Fig. 11(a d) illustrate the motional trajectories of circular nanoplate under applied voltages. It should be pointed that those solutions beyond the non-dimensional domain $[0,1]$ of dynamic governing equation have not practically physical meaning, thus can be canceled. To emphases the effect of applied voltage on the fundamental frequency, the Casimir force is not considered here; whereas other physical parameters are taken as $E^{s}=10 \mathrm{nN} / \mathrm{nm}, \tau^{s}=1.0 \mathrm{nN} / \mathrm{nm}$, $N_{m e}=1.0 \mathrm{nN} / \mathrm{nm}, \Delta T=100 \mathrm{~K}$ and $\left(e_{0} a\right)^{2}=1 \mathrm{~nm}^{2}$.

Fig. 11(a) shows the phase diagram of circular nanoplate when no voltage is applied $\left(K_{2}=0\right)$. There is one physically stable center $C$, where the dynamic system is in a rest state at this moment. When a small actuation voltage is applied on the circular nanoplate $\left(K_{2}=3.0\right)$, the phase diagram of system undergoes change, depicted in Fig. 11(b). There exist one stable focus $F$, and there are periodic orbits around the focus $F$. Homoclinic orbits originate from the other equilibrium point as one unstable saddle node $S$ and goes back to it. When the applied voltage continue to increase $\left(K_{2}=17.8\right)$, the two nodes approach to each other, shown as in Fig. 11(c). 
Finally, when the applied voltage equals to the pull-in voltage $\left(K_{2}=19.23\right)$, two nodes meet together, the stable focus $F$ eliminates and the dynamic pull-in instability of system occurs, depicted in Fig. 11(d). At this moment, the circular nanoplate loses the structural balance between elastic storing energy and electrical energy, thus collapses downwards onto the substrate.

Furthermore, Fig. 12 and Fig. 13 illustrate the pull-in instability procedure of circular nanoplate subjected to electrostatic and Casimir forces for the identical initial conditions (the initial deflection and velocity are zero), where the value of non-dimensional Casimir force is taken as $K_{4}=1$. It is observed from Fig. 12 that when no actuation voltage is applied $\left(K_{2}=0\right)$, the small deflection of circular plate occurs because the Casimir force has an attracting effect on the circular nanoplate within the nanoscale separation distance. As the applied voltage increases, the deflection gradually becomes larger; as the voltage approaches to the pull-in value $\left(K_{2}^{P I}=9.90\right)$, the deflection sharply increases. This means the system loses the dynamic stability and the pull-in instability occurs. At the pull-in voltage, the motion trajectories in the phase plane approach to the unstable saddle node, shown as in Fig. 13. As the voltage continues to increase, the circular nanoplate becomes dynamically unstable and sticks onto the substrate.

To elucidate the effect of nonlocal parameter on the dynamic pull-in instability of circular nanoplate, the time history and instable phase diagram at the pull-in voltage for different nonlocal parameters are, respectively, plotted in Fig. 14 and Fig. 15, where the surface modulus and surface residual stress are not considered $\left(E^{s}=\tau^{s}=0\right)$, and the corresponding dynamic pull-in voltage is shown as $K_{2}^{P I}=16.38$. Fig. 15 illustrates that the effect of nonlocal parameter on the instable phase diagram when the values of applied voltage increase to the pull-in voltage. It is seen from Fig. 15 that the non-dimensional velocity of dynamic system slightly decreases with the increase of nonlocal parameter; nevertheless, the pull-in voltage reduce as the nonlocal parameter increases. At the dynamic instability state, the influences of nonlocal 
parameter on velocity are not significant on the whole. The present results are consistent with those in Ref. [40], where the dynamic pull-in behaviors of graphene sheets are studied. The effect of nonlocal parameter can be explained to actually enlarge the equivalent mass, but soften the structural stiffness of nanoplate.

\section{Conclusion}

The present analytical model of 2-D circular nanoplate actuator can be harnessed in applications of nano-actuators and nano-switches in MEMS/NEMS. The switch on/off state can be precisely determined by the critical conditions of pull-in instability. The size-dependent coupling influences of elasticity deformation, electrical energy and Casimir effect are accounted for determining the dynamic behaviors and structural stability of nano-actuator system. The results indicate that the Casimir force has a softening effect on circular nanoplate, thus decreases the fundamental frequency of nano-actuator. On the other hand, the small scale parameter weakens the structural stiffness of nanoplate, so that the frequencies of the nanostructure are declined. Therefore, the values of pull-in voltage of disk-type nano-actuator are over-estimated; consequently, the excessive voltage could lead the devices to fail or damage. New results in this work may be helpful in design of electrostatically actuated nano-devices. This work aims to study the influence of small scale on the dynamic pull-in instability of circular nanoplate under the electrostatic and Casimir forces. Here, the bifurcation results around equilibrium points in Figs.11(a-d) Fig.15 have practically physical meaning for nano-devices, while other mathematical solutions beyond the non-dimensional physical range $[0,1]$ have been eliminated. The main conclusions are given by

1) Dynamic frequency and the pull-in voltage increase with the increment of surface modulus due to that surface modulus enhances the structural stiffness of circular nanoplate.

2) The pull-in voltage of circular nanoplate reduces as the surface residual stress increases. The surface residual stress decreases the frequency of circular nanoplate 
subjected to the same applied voltage.

3) Both of the pull-in voltage and frequency reduce when radial compressive mechanical loading and temperature change increase.

4) The pull-in voltage decreases as the nonlocal parameter increases and this means the nonlocal parameter has a softening effect on the rigidity of circular nanoplate.

5) At the pull-in voltage, the motion trajectories in phase diagram become closer to the unstable saddle node; by continuing to increase the voltage, the circular nanoplate loses the stable center point and becomes dynamically unstable.

\section{Acknowledgments}

The research was supported by the National Science Foundation of China under Number 11172165.

\section{Appendix A}

The associated coefficients of governing equation of electrically actuated nanoplate are defined as follows

$$
\begin{aligned}
\Psi_{0}= & \mu^{4} \int_{0}^{1} \rho^{5} \phi_{m}^{I V}(\rho) \phi_{k}(\rho) d \rho+6 \mu^{4} \int_{0}^{1} \rho^{4} \phi_{m}^{I I I}(\rho) \phi_{k}(\rho) d \rho \\
& -\mu^{2} \int_{0}^{1}\left(2 \rho^{5}-3 \mu^{2} \rho^{3}\right) \phi_{m}^{I I}(\rho) \phi_{k}(\rho) d \rho \\
& -3 \mu^{2} \int_{0}^{1}\left(2 \rho^{4}+\mu^{2} \rho^{2}\right) \phi_{m}^{I}(\rho) \phi_{k}(\rho) d \rho \\
& +\int_{0}^{1} \rho^{5} \phi_{m}(\rho) \phi_{k}(\rho) d \rho \\
\Psi_{1}= & -\left(\mathrm{K}_{2}+\mathrm{K}_{4}\right) \int_{0}^{1} \rho^{5} \phi_{m}(\rho) \phi_{k}(\rho) d \rho
\end{aligned}
$$




$$
\begin{aligned}
& \Psi_{2}=k^{s} \int_{0}^{1}\left(\rho^{5} \phi_{m}^{I V}(\rho)+2 \rho^{4} \phi_{m}^{I I I}(\rho)-\rho^{3} \phi_{m}^{I I}(\rho)+\rho^{2} \phi_{m}^{I}(\rho)\right) \phi_{k}(\rho) d \rho \\
& -\left(N_{0}+2 \tau_{0}^{s}\right) \int_{0}^{1}\left(\rho^{5} \phi_{m}^{I I}(\rho)+\rho^{4} \phi_{m}^{I}(\rho)\right) \phi_{k}(\rho) d \rho \\
& -\mu^{2}\left[k^{s}+\mu^{2}\left(N_{0}+2 \tau_{0}^{s}\right)\right] \int_{0}^{1} \rho^{5} \phi_{m}^{V I}(\rho) \phi_{k}(\rho) d \rho \\
& -7 \mu^{2}\left[k^{s}+\mu^{2}\left(N_{0}+2 \tau_{0}^{s}\right)\right] \int_{0}^{1} \rho^{4} \phi_{m}^{v}(\rho) \phi_{k}(\rho) d \rho \\
& +2 \mu^{2}\left(N_{0}+2 \tau_{0}^{s}\right) \int_{0}^{1} \rho^{5} \phi_{m}^{I V}(\rho) \phi_{k}(\rho) d \rho \\
& -\mu^{2}\left[k^{s}(3+2 v)+5 \mu^{2}\left(N_{0}+2 \tau_{0}^{s}\right)\right] \int_{0}^{1} \rho^{3} \phi_{m}^{I V}(\rho) \phi_{k}(\rho) d \rho \\
& +8 \mu^{2}\left(N_{0}+2 \tau_{0}^{s}\right) \int_{0}^{1} \rho^{4} \phi_{m}^{I I I}(\rho) \phi_{k}(\rho) d \rho \\
& +2 \mu^{2}\left[k^{s}(1+2 v)+3 \mu^{2}\left(N_{0}+2 \tau_{0}^{s}\right)\right] \int_{0}^{1} \rho^{2} \phi_{m}^{I I I}(\rho) \phi_{k}(\rho) d \rho \\
& +2 \mu^{2}\left(N_{0}+2 \tau_{0}^{s}\right) \int_{0}^{1} \rho^{3} \phi_{m}^{I I}(\rho) \phi_{k}(\rho) d \rho \\
& +3 \mu^{2}\left[k^{s}(1-2 v)-\mu^{2}\left(N_{0}+2 \tau_{0}^{s}\right)\right] \int_{0}^{1} \rho^{2} \phi_{m}^{I I}(\rho) \phi_{k}(\rho) d \rho \\
& -2 \mu^{2}\left(N_{0}+2 \tau_{0}^{s}\right) \int_{0}^{1} \rho^{2} \phi_{m}^{l}(\rho) \phi_{k}(\rho) d \rho \\
& -3 \mu^{2}\left[k^{s}(1-2 v)-\mu^{2}\left(N_{0}+2 \tau_{0}^{s}\right)\right] \int_{0}^{1} \rho^{2} \phi_{m}^{I}(\rho) \phi_{k}(\rho) d \rho \\
& -\left(2 K_{2}+4 K_{4}\right) \int_{0}^{1}\left[\rho^{5}-3 \mu^{2}\left(2 \rho^{2}+\mu^{2}\right) \rho^{2} \phi_{m}^{I}(\rho)\right. \\
& \left.+\mu^{2}\left(2 \rho^{2}-3 \mu^{2}\right) \rho^{3} \phi_{m}^{I I}(\rho)+6 \mu^{4} \rho^{4} \phi_{m}^{I I I}(\rho)+\mu^{4} \rho^{5} \phi_{m}^{I V}(\rho)\right] \phi_{k}(\rho) d \rho \\
& \Psi_{3}=-\left(3 K_{2}+10 K_{4}\right) \int_{0}^{1}\left[\rho^{5} \phi_{m}(\rho) \phi_{k}(\rho)-6 \mu^{2}\left(2 \rho^{2}+\mu^{2}\right) \rho^{2} \phi_{m}^{I}(\rho) \phi_{k}(\rho)\right. \\
& +\mu^{2}\left(2 \rho^{2}-3 \mu^{2}\right) \rho^{3}\left(2 \phi_{m}^{I}(\rho) \phi_{k}^{I}(\rho)+2 \phi_{m}(\rho) \phi_{k}^{I I}(\rho)\right) \\
& +6 \mu^{4} \rho^{4}\left(6 \phi_{m}^{I}(\rho) \phi_{k}^{I I}(\rho)+2 \phi_{m}(\rho) \phi_{k}^{I I I}(\rho)\right) \\
& \left.+\mu^{4} \rho^{5}\left(6 \phi_{m}^{I I}(\rho) \phi_{k}^{I I}(\rho)+8 \phi_{m}(\rho) \phi_{k}^{I I I}(\rho)+2 \phi_{m}(\rho) \phi_{k}^{I V}(\rho)\right)\right] \phi_{l}(\rho) d \rho \\
& \Psi_{4}=-\left(4 K_{2}+20 K_{4}\right) \int_{0}^{1}\left[\rho^{5} \phi_{m}(\rho) \phi_{k}(\rho) \phi_{l}(\rho)-9 \mu^{2}\left(2 \rho^{2}+\mu^{2}\right) \rho^{2} \phi_{m}(\rho) \phi_{k}(\rho) \phi_{l}^{I}(\rho)\right. \\
& +\mu^{2}\left(2 \rho^{2}-3 \mu^{2}\right) \rho^{3}\left(6 \phi_{m}(\rho) \phi_{k}^{I}(\rho) \phi_{l}^{I}(\rho)+3 \phi_{m}(\rho) \phi_{k}(\rho) \phi_{l}^{I I}(\rho)\right) \\
& +6 \mu^{4} \rho^{4}\left(6 \phi_{m}^{I}(\rho) \phi_{k}^{I}(\rho) \phi_{l}^{I}(\rho)+18 \phi_{m}(\rho) \phi_{k}^{I}(\rho) \phi_{l}^{I I}(\rho)+3 \phi_{m}(\rho) \phi_{k}(\rho) \phi_{l}^{I I I}(\rho)\right) \\
& +\mu^{4} \rho^{5}\left(36 \phi_{m}^{I}(\rho) \phi_{k}^{I}(\rho) \phi_{l}^{I I}(\rho)+18 \phi_{m}(\rho) \phi_{k}^{I I}(\rho) \phi_{l}^{I I}(\rho)+24 \phi_{m}(\rho) \phi_{k}^{I}(\rho) \phi_{l}^{I I I}(\rho)\right. \\
& \left.\left.+3 \phi_{m}(\rho) \phi_{k}(\rho) \phi_{l}^{I V}(\rho)\right)\right] \phi_{n}(\rho) d \rho
\end{aligned}
$$




$$
\begin{aligned}
\Psi_{5}= & -\left(5 K_{2}+35 K_{4}\right) \int_{0}^{1}\left[\rho^{5} \phi_{m}(\rho) \phi_{k}(\rho) \phi_{l}(\rho) \phi_{n}(\rho)\right. \\
& -12 \mu^{2}\left(2 \rho^{2}+\mu^{2}\right) \rho^{2} \phi_{m}(\rho) \phi_{k}(\rho) \phi_{l}(\rho) \phi_{n}^{I}(\rho) \\
& +\mu^{2}\left(2 \rho^{2}-3 \mu^{2}\right) \rho^{3}\left(12 \phi_{m}(\rho) \phi_{k}(\rho) \phi_{l}^{I}(\rho) \phi_{n}^{I}(\rho)\right. \\
& \left.+4 \phi_{m}(\rho) \phi_{k}(\rho) \phi_{l}(\rho) \phi_{n}^{I I}(\rho)\right)+6 \mu^{4} \rho^{4}\left(24 \phi_{m}^{I}(\rho) \phi_{k}^{I}(\rho) \phi_{l}^{I}(\rho) \phi_{n}(\rho)\right. \\
& \left.+36 \phi_{m}(\rho) \phi_{k}(\rho) \phi_{l}^{I}(\rho) \phi_{n}^{I I}(\rho)+4 \phi_{m}(\rho) \phi_{k}(\rho) \phi_{l}(\rho) \phi_{n}^{I I I}(\rho)\right) \\
& +\mu^{4} \rho^{5}\left(24 \phi_{m}^{I}(\rho) \phi_{k}^{I}(\rho) \phi_{l}^{I}(\rho) \phi_{n}^{I}(\rho)+144 \phi_{m}(\rho) \phi_{k}^{I}(\rho) \phi_{l}^{I}(\rho) \phi_{n}^{I I}(\rho)\right. \\
& +36 \phi_{m}(\rho) \phi_{k}(\rho) \phi_{l}^{I I}(\rho) \phi_{n}^{I I}(\rho)+48 \phi_{m}(\rho) \phi_{k}(\rho) \phi_{l}^{I}(\rho) \phi_{n}^{I I I}(\rho) \\
& \left.\left.+4 \phi_{m}(\rho) \phi_{k}(\rho) \phi_{l}(\rho) \phi_{n}^{I V}(\rho)\right)\right] \phi_{j}(\rho) d \rho
\end{aligned}
$$

\section{Appendix B}

\section{The flowchart of algorithm realization}

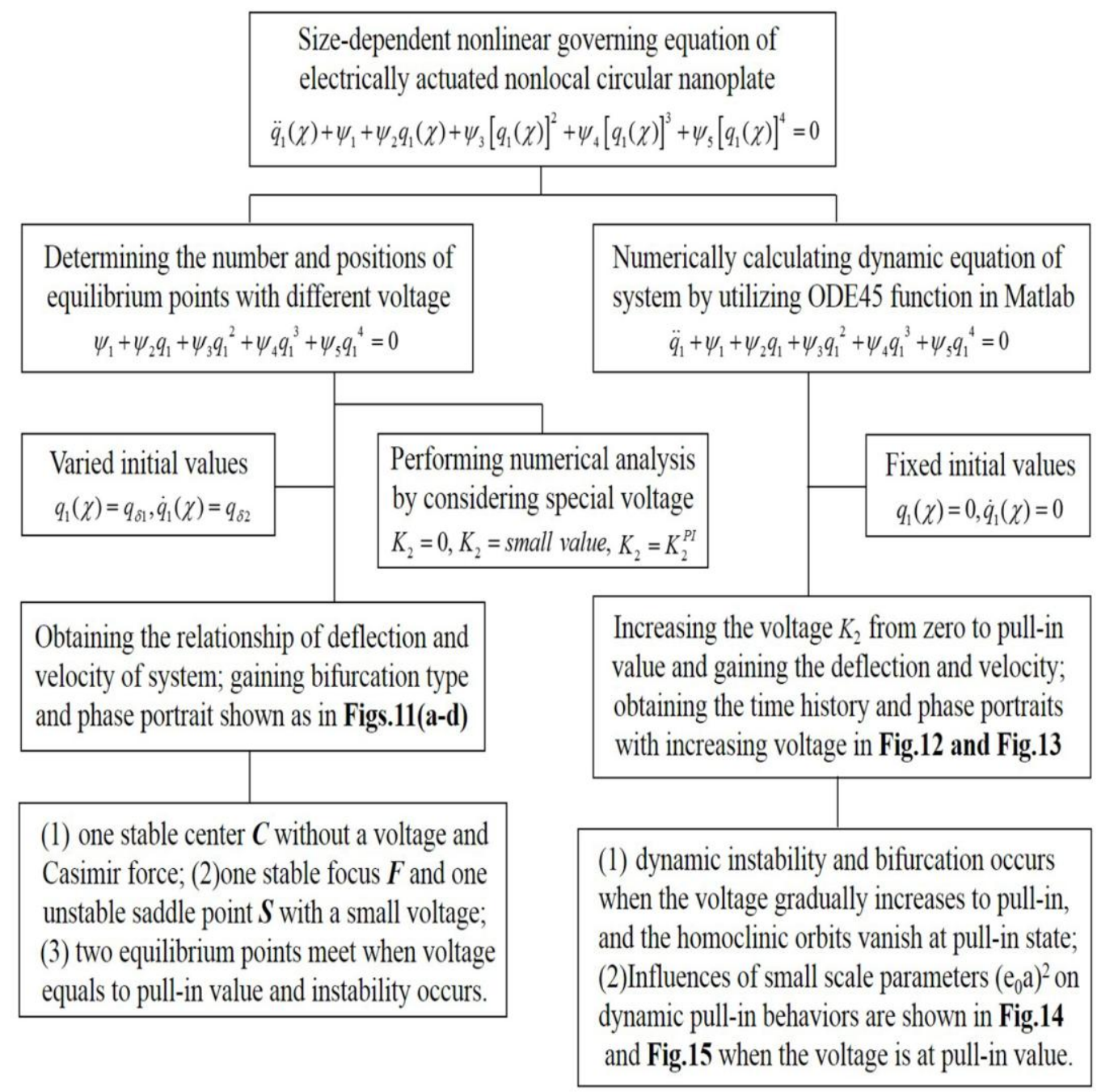




\section{References}

[1] S. Souayeh, N. Kacem, Computational models for large amplitude nonlinear vibrations of electrostatically actuated carbon nanotube-based mass sensors, Sens. Actuators, A 208 (2014) 10-20.

[2] S. Kruss, A.J. Hilmer, J. Zhang, N.F. Reuel, B. Mu, M.S. Strano, Carbon nanotubes as optical biomedical sensors, Adv. Drug Delivery Rev. , 65 (2013) 1933-1950.

[3] M. Ren, J. Huang, H. Cai, J.M. Tsai, J. Zhou, Z. Liu, Z. Suo, A.-Q. Liu, Nano-optomechanical actuator and pull-back instability, ACS nano, 7 (2013) 1676-1681.

[4] H.M. Sedighi, F. Daneshmand, M. Abadyan, Dynamic instability analysis of electrostatic functionally graded doubly-clamped nano-actuators, Compos. Struct. , 124 (2015) 55-64.

[5] W. Yang, X. Wang, Nonlinear pull-in instability of carbon nanotubes reinforced nano-actuator with thermally corrected Casimir force and surface effect, Int. J. Mech. Sci. , (2016).

[6] S. Malihi, Y.T. Beni, H. Golestanian, Analytical modeling of dynamic pull-in instability behavior of torsional nano/micromirrors under the effect of Casimir force, Optik-International Journal for Light and Electron Optics, 127 (2016) 4426-4437.

[7] M. Shojaeian, Y.T. Beni, Size-dependent electromechanical buckling of functionally graded electrostatic nano-bridges, Sens. Actuators, A 232 (2015) 49-62.

[8] D.I. Caruntu, I. Martinez, Reduced order model of parametric resonance of electrostatically actuated MEMS cantilever resonators, Int. J. Non Linear Mech. , 66 (2014) 28-32.

[9] M.H. Ghayesh, H. Farokhi, M. Amabili, Nonlinear behaviour of electrically actuated MEMS resonators, Int. J. Eng Sci 71 (2013) 137-155.

[10] J.S. Bunch, A.M. Van Der Zande, S.S. Verbridge, I.W. Frank, D.M. Tanenbaum, J.M. Parpia, H.G. Craighead, P.L. McEuen, Electromechanical resonators from graphene sheets, Science, 315 (2007) 490-493.

[11] O. Loh, X. Wei, J. Sullivan, L.E. Ocola, R. Divan, H.D. Espinosa, Carbon - Carbon Contacts for Robust Nanoelectromechanical Switches, Adv. Mater. , 24 (2012) 2463-2468.

[12] T. Mousavi, S. Bornassi, H. Haddadpour, The effect of small scale on the pull-in instability of nano-switches using DQM, Int. J. Solids Struct. , 50 (2013) 1193-1202.

[13] O.Y. Loh, H.D. Espinosa, Nanoelectromechanical contact switches, Nat. Nanotechnol. , 7 (2012) 283-295.

[14] A. Ramezani, Stability analysis of electrostatic nanotweezers, Physica E 43 (2011) 1783-1791.

[15] A. Chowdhury, I. Yeo, V. Tsvirkun, F. Raineri, G. Beaudoin, I. Sagnes, R. Raj, I. Robert-Philip, R. Braive, Superharmonic resonances in a two-dimensional non-linear photonic-crystal nano-electro-mechanical oscillator, Appl. Phys. Lett. , 108 (2016) 163102.

[16] X. Feng, C. White, A. Hajimiri, M.L. Roukes, A self-sustaining ultrahigh-frequency nanoelectromechanical oscillator, Nat. Nanotechnol. , 3 (2008) 342-346.

[17] M. Mojahedi, M.T. Ahmadian, K. Firoozbakhsh, Effects of Casimir and Van Der Waals Forces on the Pull-in Instability of the Nonlinear Micro and Nano-Bridge Gyroscopes, Int. J. Struct. Stab. Dyn. , 14 (2014) 1350059.

[18] W.-M. Zhang, H. Yan, Z.-K. Peng, G. Meng, Electrostatic pull-in instability in MEMS/NEMS: A review, Sens. Actuators, A 214 (2014) 187-218.

[19] J.-G. Guo, Y.-P. Zhao, Dynamic stability of electrostatic torsional actuators with van der Waals effect, Int. J. Solids Struct. , 43 (2006) 675-685.

[20] H.M. Sedighi, F. Daneshmand, M. Abadyan, Modeling the effects of material properties on the pull 
- in instability of nonlocal functionally graded nano - actuators, ZAMM - Journal of Applied Mathematics and Mechanics/Zeitschrift für Angewandte Mathematik und Mechanik, (2015).

[21] A.W. Rodriguez, F. Capasso, S.G. Johnson, The Casimir effect in microstructured geometries, Nat. Photonics 5(2011) 211-221.

[22] Y.T. Beni, I. Karimipöur, M. Abadyan, Modeling the effect of intermolecular force on the size-dependent pull-in behavior of beam-type NEMS using modified couple stress theory, J. Mech. Sci. Technol. , 28 (2014) 3749-3757.

[23] Y.T. Beni, I. Karimipour, M. Abadyan, Modeling the instability of electrostatic nano-bridges and nano-cantilevers using modified strain gradient theory, Applied Mathematical Modelling, 39 (2015) 2633-2648.

[24] Y.T. Beni, A. Vahdati, M. Abadyan, Using ALE-FEM to simulate the instability of beam-type nano-actuator in the presence of electrostatic field and dispersion forces, Iranian Journal of Science and Technology. Transactions of Mechanical Engineering, 37 (2013) 1.

[25] M. Shojaeian, Y.T. Beni, H. Ataei, Electromechanical buckling of functionally graded electrostatic nanobridges using strain gradient theory, Acta Astronaut. , 118 (2016) 62-71.

[26] H.B. Casimir, On the attraction between two perfectly conducting plates, in: Proceedings of the KNAW, 1948, pp. 793-795.

[27] W.-H. Lin, Y.-P. Zhao, Nonlinear behavior for nanoscale electrostatic actuators with Casimir force, Chaos, Solitons Fractals 23 (2005) 1777-1785.

[28] R. Batra, M. Porfiri, D. Spinello, Reduced-order models for microelectromechanical rectangular and circular plates incorporating the Casimir force, Int. J. Solids Struct. , 45 (2008) 3558-3583.

[29] R. Agrawal, B. Peng, E.E. Gdoutos, H.D. Espinosa, Elasticity size effects in ZnO nanowires- a combined experimental-computational approach, Nano Lett. , 8 (2008) 3668-3674.

[30] R.E. Miller, V.B. Shenoy, Size-dependent elastic properties of nanosized structural elements, Nanotechnology, 11 (2000) 139.

[31] A. Eringen, Theory of Nonlocal Elasticity and Some Applications, in, DTIC Document, 1984.

[32] A.C. Eringen, D. Edelen, On nonlocal elasticity, Int. J. Eng Sci 10 (1972) 233-248.

[33] J. Peddieson, G.R. Buchanan, R.P. McNitt, Application of nonlocal continuum models to nanotechnology, Int. J. Eng Sci 41 (2003) 305-312.

[34] J.N. Reddy, Nonlocal theories for bending, buckling and vibration of beams, Int. J. Eng Sci 45 (2007) 288-307.

[35] S. Pradhan, J. Phadikar, Nonlocal elasticity theory for vibration of nanoplates, Journal of Sound and Vibration, 325 (2009) 206-223.

[36] A.C. Eringen, On differential equations of nonlocal elasticity and solutions of screw dislocation and surface waves, J. Appl. Phys. , 54 (1983) 4703-4710.

[37] Y. Zhang, C. Wang, V. Tan, Assessment of Timoshenko beam models for vibrational behavior of single-walled carbon nanotubes using molecular dynamics, Adv. Appl. Math. Mech, 1 (2009) 89-106.

[38] H.-S. Shen, Y.-M. Xu, C.-L. Zhang, Prediction of nonlinear vibration of bilayer graphene sheets in thermal environments via molecular dynamics simulations and nonlocal elasticity, Computer Methods in Applied Mechanics and Engineering, 267 (2013) 458-470.

[39] A.G. Arani, M. Ghaffari, A. Jalilvand, R. Kolahchi, Nonlinear nonlocal pull-in instability of boron nitride nanoswitches, Acta Mech. , 224 (2013) 3005-3019.

[40] K. Wang, B. Wang, S. Zeng, Small scale effect on the pull-in instability and vibration of graphene sheets, Microsyst. Technol. , (2016) 1-9. 
[41] R. Dingreville, J. Qu, M. Cherkaoui, Surface free energy and its effect on the elastic behavior of nano-sized particles, wires and films, J. Mech. Phys. Solids 53 (2005) 1827-1854.

[42] F. Fischer, T. Waitz, D. Vollath, N. Simha, On the role of surface energy and surface stress in phase-transforming nanoparticles, Prog. Mater Sci. , 53 (2008) 481-527.

[43] M. Keivani, A. Koochi, H.M. Sedighi, A. Abadian, M. Abadyan, A Nonlinear Model for Incorporating the Coupled Effects of Surface Energy and Microstructure on the Electromechanical Stability of NEMS, Arabian Journal for Science and Engineering, (2016) 1-14.

[44] M. Keivani, A. Koochi, N. Abadian, M. Rezaei, M. Abadyan, Static and dynamic instability of nanowire-fabricated nanoelectromechanical systems: effects of flow damping, van de Waals force, surface energy and microstructure, Can. J. Phys. , 94 (2016) 594-603.

[45] M.E. Gurtin, A.I. Murdoch, A continuum theory of elastic material surfaces, Arch. Ration. Mech. Anal. , 57 (1975) 291-323.

[46] M.E. Gurtin, A.I. Murdoch, Surface stress in solids, Int. J. Solids Struct. , 14 (1978) 431-440.

[47] R. Ansari, R. Gholami, M.F. Shojaei, V. Mohammadi, S. Sahmani, Surface stress effect on the pull-in instability of circular nanoplates, Acta Astronaut. , 102 (2014) 140-150.

[48] W.D. Yang, X. Wang, C.Q. Fang, G. Lu, Electromechanical coupling characteristics of carbon nanotube reinforced cantilever nano-actuator, Sens. Actuators, A 220 (2014) 178-187.

[49] W. Yang, X. Wang, C. Fang, Pull-in instability of carbon nanotube-reinforced nano-switches considering scale, surface and thermal effects, Composites Part B: Engineering, 82 (2015) 143-151.

[50] S. Sahmani, M. Bahrami, Nonlocal plate model for dynamic pull-in instability analysis of circular higher-order shear deformable nanoplates including surface stress effect, J. Mech. Sci. Technol. , 29 (2015) 1151-1161.

[51] J.-H. He, Homotopy perturbation method: a new nonlinear analytical technique, Applied Mathematics and computation, 135 (2003) 73-79.

[52] J.-H. He, Recent development of the homotopy perturbation method, Topological Methods in Nonlinear Analysis, 31 (2008) 205-209.

[53] M. Mojahedi, M.M. Zand, M. Ahmadian, Static pull-in analysis of electrostatically actuated microbeams using homotopy perturbation method, Applied Mathematical Modelling, 34 (2010) 1032-1041.

[54] M. Sheikholeslami, H. Ashorynejad, D. Ganji, A. Yıldırım, Homotopy perturbation method for three-dimensional problem of condensation film on inclined rotating disk, Scientia Iranica, 19 (2012) 437-442.

[55] A.C. Eringen, On differential equations of nonlocal elasticity and solutions of screw dislocation and surface waves, J. Appl. Phys. , 54 (1983) 4703.

[56] S.R. Asemi, A. Farajpour, Decoupling the nonlocal elasticity equations for thermo-mechanical vibration of circular graphene sheets including surface effects, Physica E 60 (2014) 80-90.

[57] J.N. Reddy, Theory and analysis of elastic plates and shells, CRC press, 2006.

[58] W. Duan, C.M. Wang, Exact solutions for axisymmetric bending of micro/nanoscale circular plates based on nonlocal plate theory, Nanotechnology, 18 (2007) 385704.

[59] G.W. Vogl, A.H. Nayfeh, A reduced-order model for electrically actuated clamped circular plates, in: ASME 2003 International Design Engineering Technical Conferences and Computers and Information in Engineering Conference, American Society of Mechanical Engineers, 2003, pp. 1867-1874.

[60] M.M. Zand, M.T. Ahmadian, Application of homotopy analysis method in studying dynamic pull-in instability of microsystems, Mechanics Research Communications, 36 (2009) 851-858. 
[61] P.M. Osterberg, Electrostatically actuated microelectromechanical test structures for material property measurement, in, Massachusetts Institute of Technology, 1995.

[62] P. Raback, A. Pursula, Finite element simulation of the electromechanical pull-in phenomenon, in: European Congress on Computational Methods in Applied Sciences and Engineering ECCOMAS 2004, 2004, pp. 24-28.

[63] K. Rashvand, G. Rezazadeh, H. Mobki, M.H. Ghayesh, On the size-dependent behavior of a capacitive circular micro-plate considering the variable length-scale parameter, Int. J. Mech. Sci. , 77 (2013) 333-342.

\section{Caption of Figures}

Fig. 1 Schematic of electrically actuated circular nanoplate with surface layer for (a) top view and (b) side view.

Fig. 2. The influences of surface elasticity modulus on non-dimensional frequency and voltage $\left(\tau^{s}=1.0 \mathrm{nN} / \mathrm{nm}, \Delta T=100 \mathrm{~K}, N_{m e}=1 \mathrm{nN} / \mathrm{nm}, \quad\left(e_{0} a\right)^{2}=1 \mathrm{~nm}^{2}, K_{4}=1\right.$, $A=0.2$ ).

Fig. 3. The influences of surface residual stress on non-dimensional frequency and voltage $\left(E^{s}=1.0 \mathrm{nN} / \mathrm{nm}, \Delta T=100 \mathrm{~K}, N_{m e}=1 \mathrm{nN} / \mathrm{nm}, \quad\left(e_{0} a\right)^{2}=1 \mathrm{~nm}^{2}, K_{4}=1\right.$, $A=0.2$ ).

Fig. 4. The influences of axial mechanical loading on non-dimensional frequency and voltage $\left(E^{s}=\tau^{s}=1.0 \mathrm{nN} / \mathrm{nm}, \Delta T=100 \mathrm{~K},\left(e_{0} a\right)^{2}=1 \mathrm{~nm}^{2}, K_{4}=1, A=0.2\right)$.

Fig. 5. The influences of temperature change on non-dimensional frequency and voltage $\left(E^{s}=\tau^{s}=1.0 \mathrm{nN} / \mathrm{nm}, \Delta T=100 \mathrm{~K}, \quad N_{m e}=1 \mathrm{nN} / \mathrm{nm}, \quad\left(e_{0} a\right)^{2}=1 \mathrm{~nm}^{2}\right.$, $\left.K_{4}=1, \quad A=0.2\right)$.

Fig. 6. The influences of nonlocal parameter on non-dimensional frequency and voltage $\left(E^{s}=\tau^{s}=1.0 \mathrm{nN} / \mathrm{nm}, N_{m e}=1 \mathrm{nN} / \mathrm{nm}, \Delta T=100 \mathrm{~K}, K_{4}=1, A=0.2\right)$.

Fig. 7. The coupling effect of surface elasticity and residual stress on dynamic frequency $\left(K_{2}=5, N_{m e}=\Delta T=0, K_{4}=1, A=0.2\right)$.

Fig. 8. The coupling effect of axial mechanical loading and temperature change on dynamic frequency $\left(K_{2}=5, E^{s}=\tau^{s}=0, K_{4}=1, A=0.2\right)$.

Fig. 9. The influences of ratio of radius-to-thickness on non-dimensional frequency and voltage $\left(E^{s}=\tau^{s}=1.0 \mathrm{nN} / \mathrm{nm}, N_{m e}=1 \mathrm{nN} / \mathrm{nm},\left(e_{0} a\right)^{2}=1 \mathrm{~nm}^{2}, \Delta T=100 \mathrm{~K}\right.$, $K_{4}=1, A=0.2$ ). 
Fig. 10. The effect of nonlocal parameter on non-dimensional frequency for different ratios of radius-to-thickness $\left(K_{2}=0, E^{s}=\tau^{s}=1.0 \mathrm{nN} / \mathrm{nm}, N_{m e}=1 \mathrm{nN} / \mathrm{nm}\right.$, $\left.\Delta T=100 \mathrm{~K}, \quad K_{4}=1, A=0.2\right)$.

Fig. 11. The transition history of equilibrium points in phase diagram of circular nanoplate when (a) without applied voltage $\left(K_{2}=0\right)$ and Casimir force, (b) small voltage $\left(K_{2}=3.0\right)$, (c) near pull-in voltage $\left(K_{2}=17.8\right)$, and (d) at the pull-in state $\left(K_{2}=19.23\right)$.

Fig. 12. The time history responses of circular nanoplate under applied voltage and Casimir force $\left(E^{s}=10.605 \mathrm{nN} / \mathrm{nm}, \tau^{s}=1.0 \mathrm{nN} / \mathrm{nm}, \Delta T=100 \mathrm{~K}, N_{m e}=1 \mathrm{nN} / \mathrm{nm}\right.$, $\left.\left(e_{0} a\right)^{2}=1 \mathrm{~nm}^{2}, K_{4}=1\right)$.

Fig. 13. The phase diagram of circular nanoplate under applied voltage and Casimir force $\left(E^{s}=10.605 \mathrm{nN} / \mathrm{nm}, \quad \tau^{s}=1.0 \mathrm{nN} / \mathrm{nm}, \Delta T=100 \mathrm{~K}, \quad N_{m e}=1 \mathrm{nN} / \mathrm{nm}\right.$, $\left.\left(e_{0} a\right)^{2}=1 \mathrm{~nm}^{2}, K_{4}=1\right)$.

Fig. 14. The small scale effect on time history response of circular nanoplate at the dynamic pull-in state ( $\left.E^{s}=\tau^{s}=0, N_{m e}=\Delta T=0, K_{4}=1\right)$.

Fig. 15. The small scale effect on phase diagram of circular nanoplate at the dynamic pull-in state $\left(E^{s}=\tau^{s}=0, N_{m e}=\Delta T=0, K_{4}=1\right)$.

Table 1. Influence of Casimir force on deflection by HPM and numerical method.

Table 2. Geometrical and material parameters of micro-plate for validation.

Table 3. Comparison of dynamic pull-in voltages from various methods.

Table 4. The material and geometrical parameters of circular nanoplate. 
(a) Top view

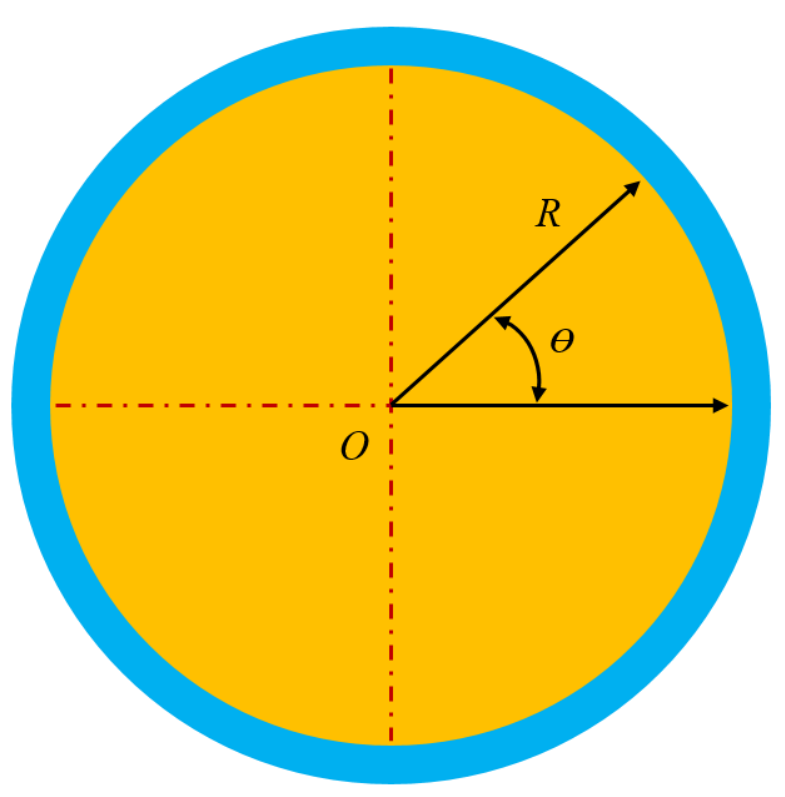

(b) Side view

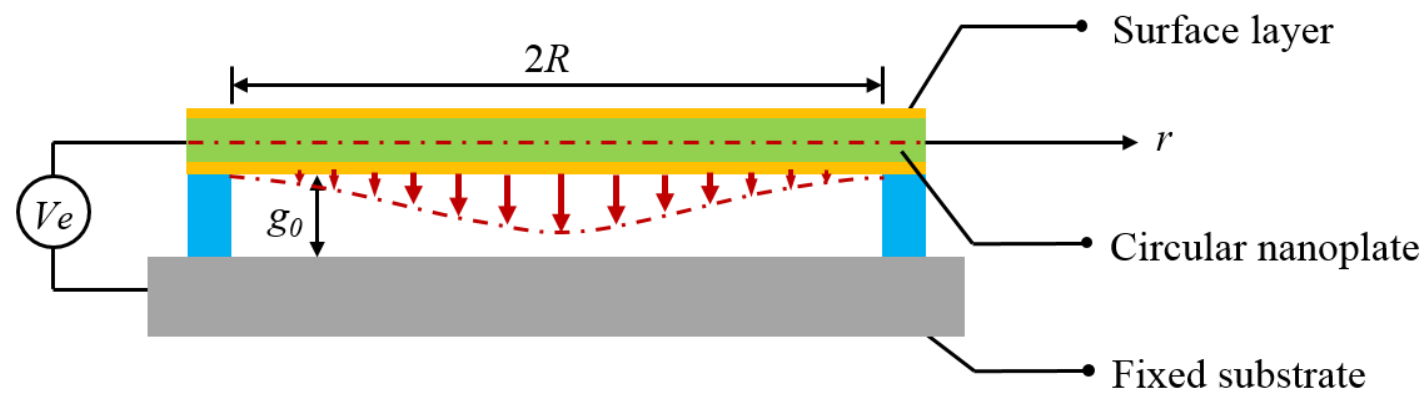

Fig. 1 Schematic of electrically actuated circular nanoplate with surface layer for (a) top view and (b) side view. 


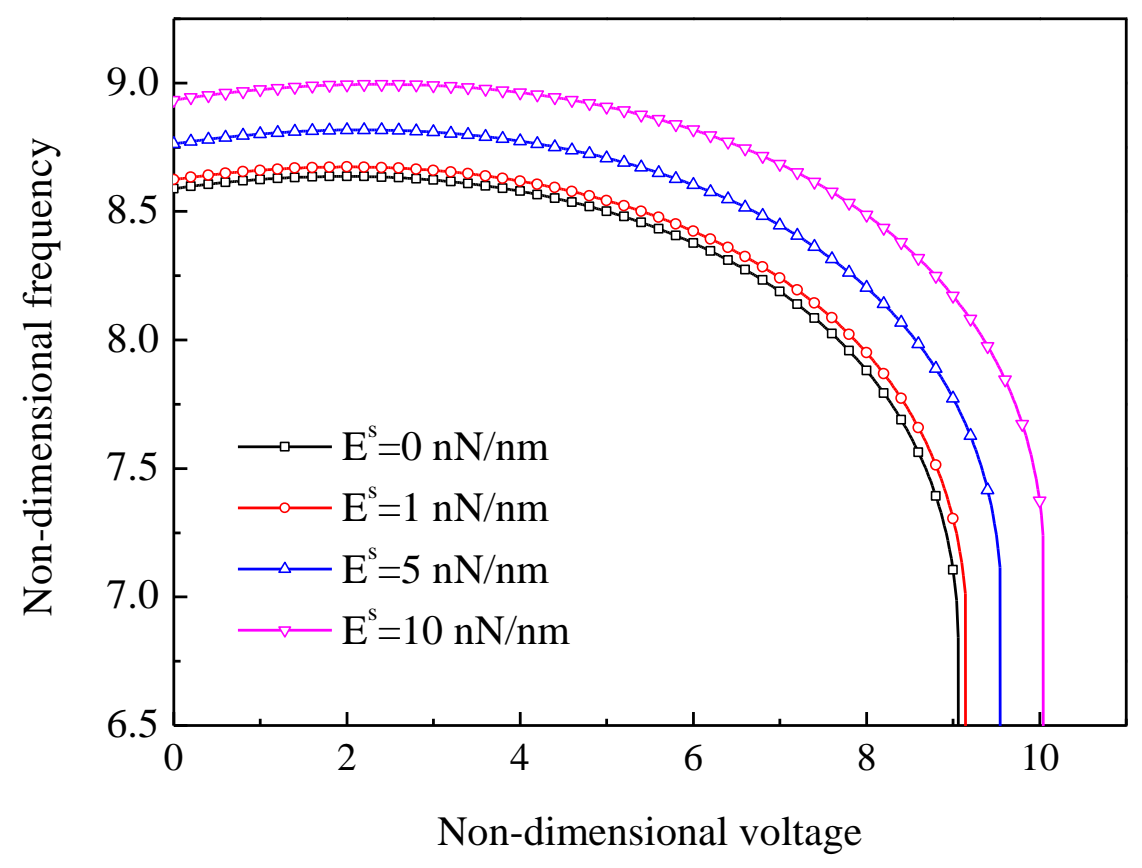

Fig. 2. The influences of surface elasticity modulus on non-dimensional frequency and voltage $\left(\tau^{s}=1.0 \mathrm{nN} / \mathrm{nm}, \Delta T=100 \mathrm{~K}, N_{m e}=1 \mathrm{nN} / \mathrm{nm},\left(e_{0} a\right)^{2}=1 \mathrm{~nm}^{2}, K_{4}=1, A=0.2\right)$.

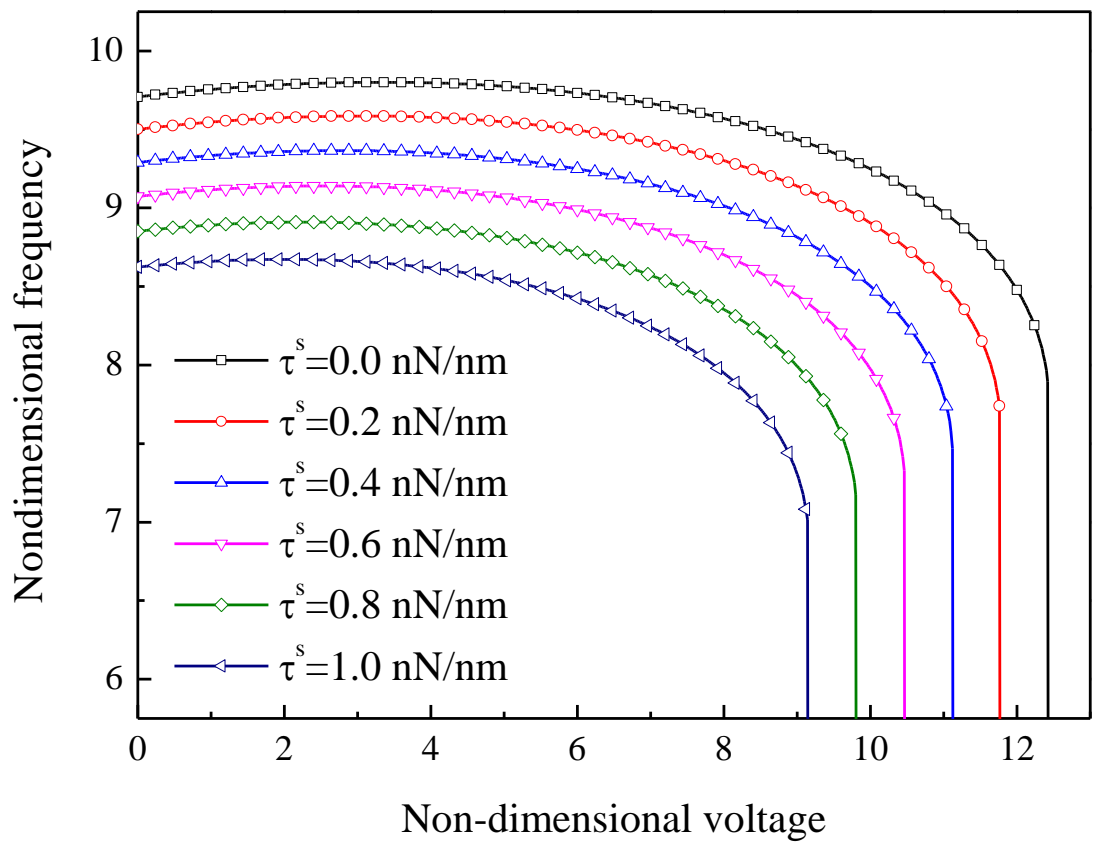

Fig. 3. The influences of surface residual stress on non-dimensional frequency and voltage $\left(E^{s}=1.0 \mathrm{nN} / \mathrm{nm}, \Delta T=100 \mathrm{~K}, N_{m e}=1 \mathrm{nN} / \mathrm{nm}, \quad\left(e_{0} a\right)^{2}=1 \mathrm{~nm}^{2}, K_{4}=1, A=0.2\right)$. 


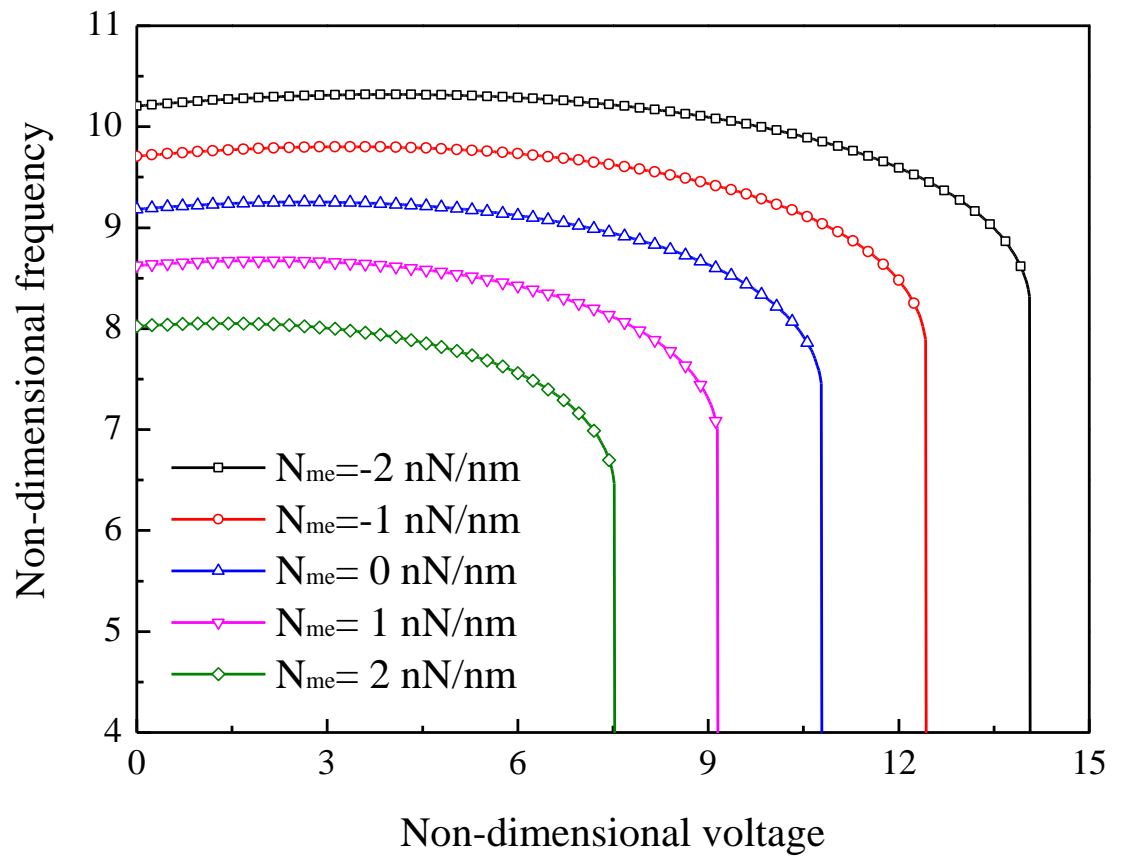

Fig. 4. The influences of axial mechanical loading on non-dimensional frequency and voltage $\left(E^{s}=\tau^{s}=1.0 \mathrm{nN} / \mathrm{nm}, \Delta T=100 \mathrm{~K},\left(e_{0} a\right)^{2}=1 \mathrm{~nm}^{2}, K_{4}=1, A=0.2\right)$.

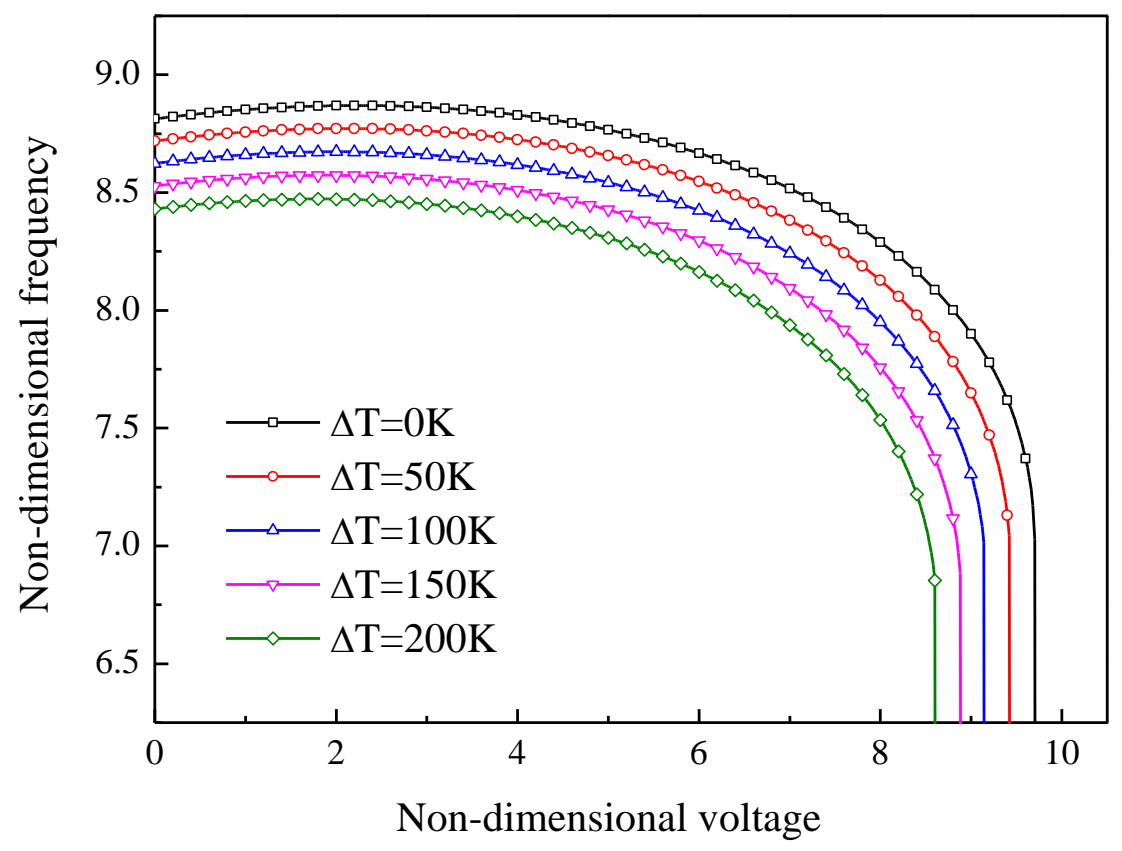

Fig. 5. The influences of temperature change on non-dimensional frequency and voltage $\left(E^{s}=\tau^{s}=1.0 \mathrm{nN} / \mathrm{nm}, \Delta T=100 \mathrm{~K}, N_{m e}=1 \mathrm{nN} / \mathrm{nm},\left(e_{0} a\right)^{2}=1 \mathrm{~nm}^{2}, K_{4}=1, A=0.2\right)$. 


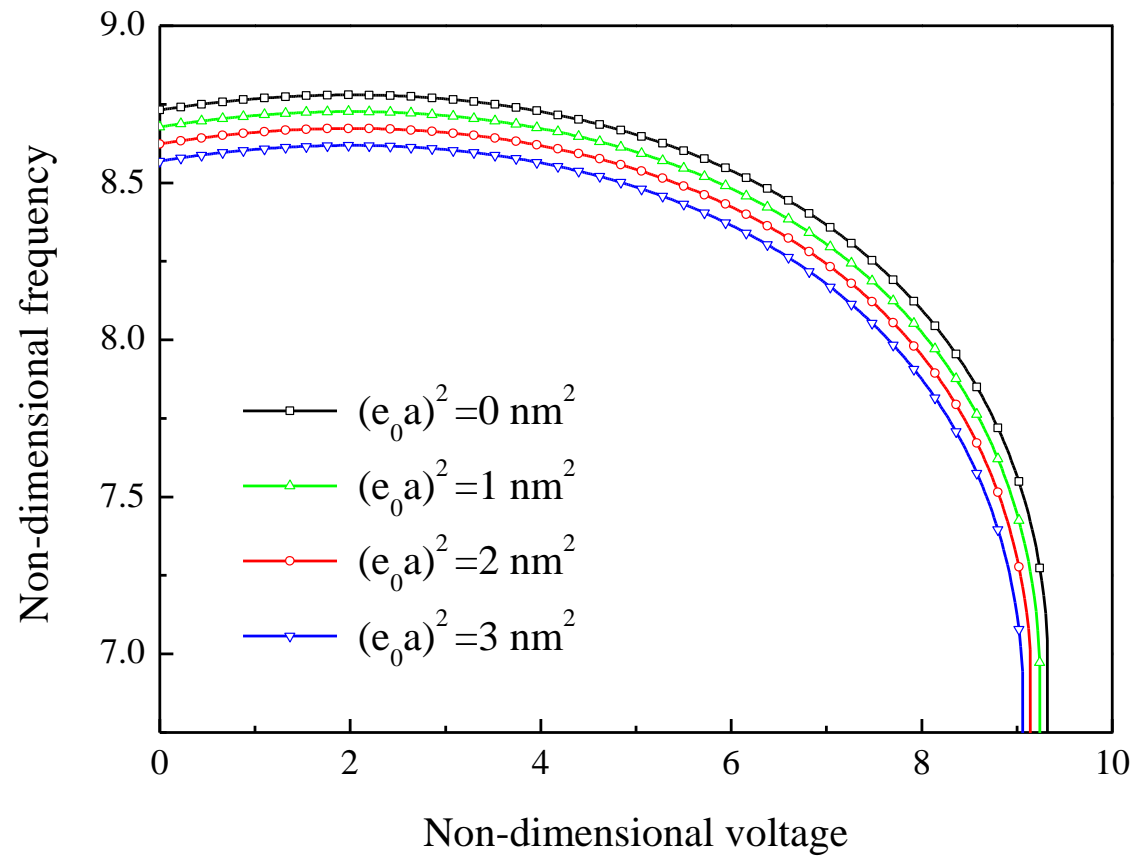

Fig. 6. The influences of nonlocal parameter on non-dimensional frequency and voltage $\left(E^{s}=\tau^{s}=1.0 \mathrm{nN} / \mathrm{nm}, N_{m e}=1 \mathrm{nN} / \mathrm{nm}, \Delta T=100 \mathrm{~K}, K_{4}=1, A=0.2\right)$.

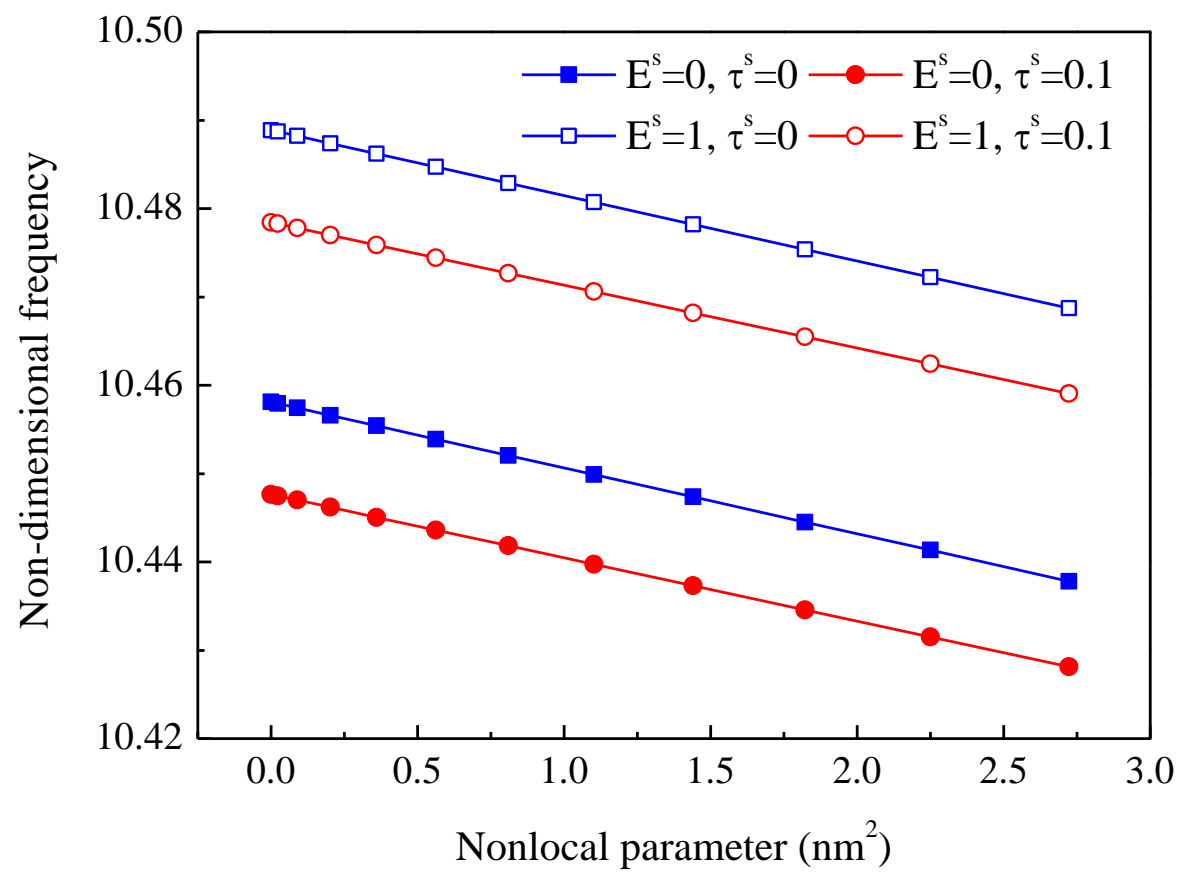

Fig. 7. The coupling effect of surface elasticity and residual stress on dynamic

$$
\text { frequency }\left(K_{2}=5, N_{m e}=\Delta T=0, K_{4}=1, A=0.2\right) \text {. }
$$




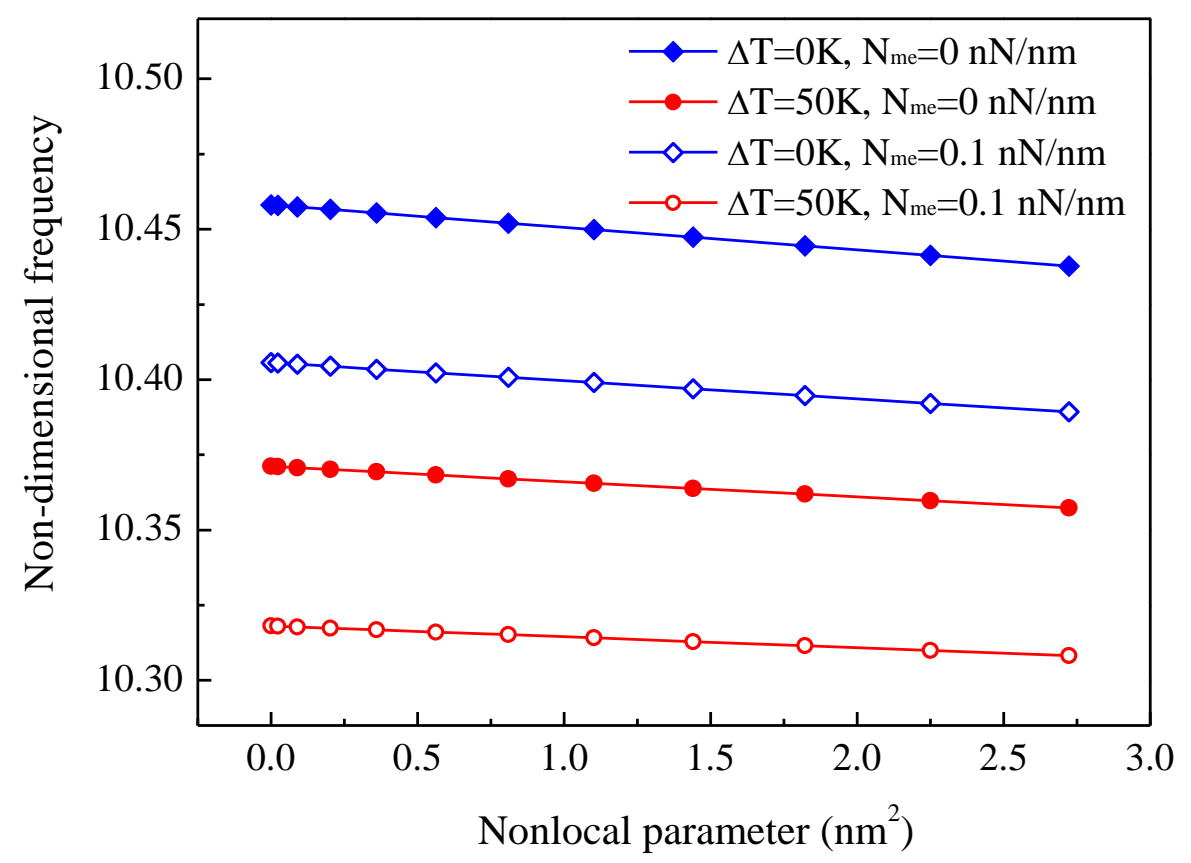

Fig. 8. The coupling effect of axial mechanical loading and temperature change on dynamic frequency $\left(K_{2}=5, E^{s}=\tau^{s}=0, K_{4}=1, A=0.2\right)$.

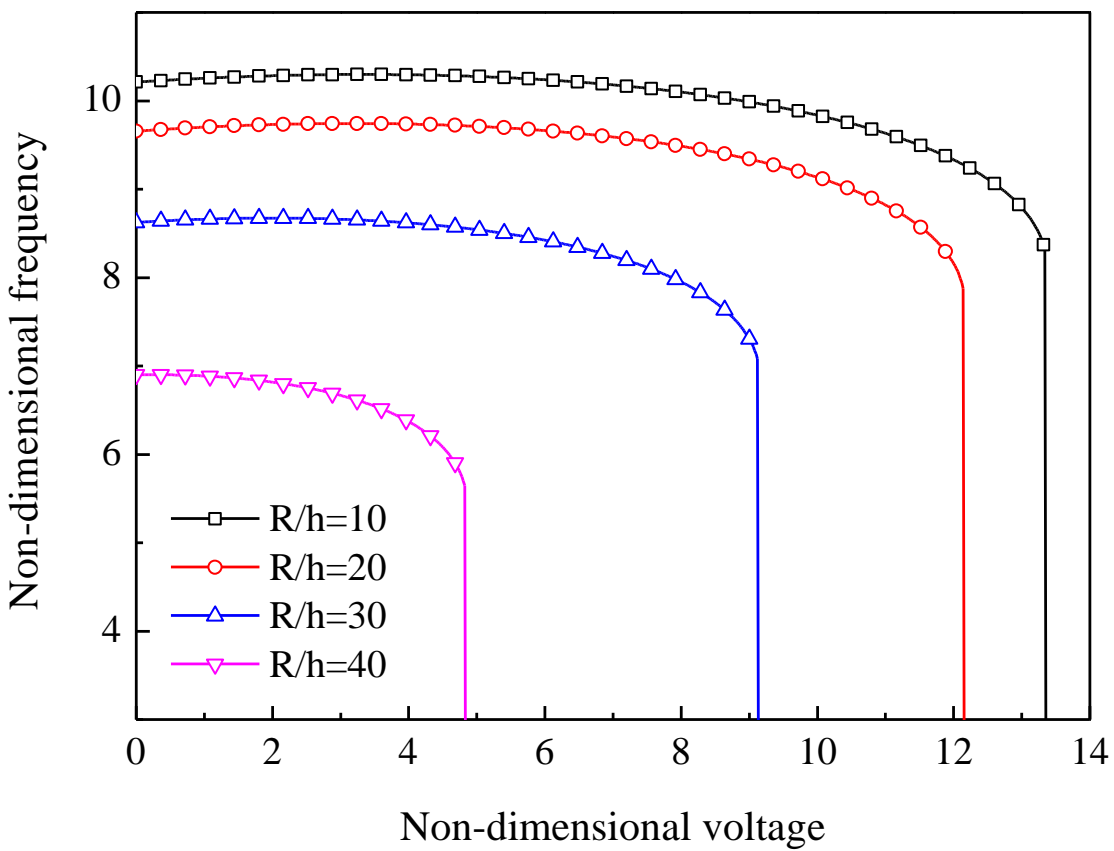

Fig. 9. The influences of ratio of radius-to-thickness on non-dimensional frequency and voltage $\left(E^{s}=\tau^{s}=1.0 \mathrm{nN} / \mathrm{nm}, N_{m e}=1 \mathrm{nN} / \mathrm{nm},\left(e_{0} a\right)^{2}=1 \mathrm{~nm}^{2}, \Delta T=100 \mathrm{~K}, K_{4}=1, A=0.2\right)$. 


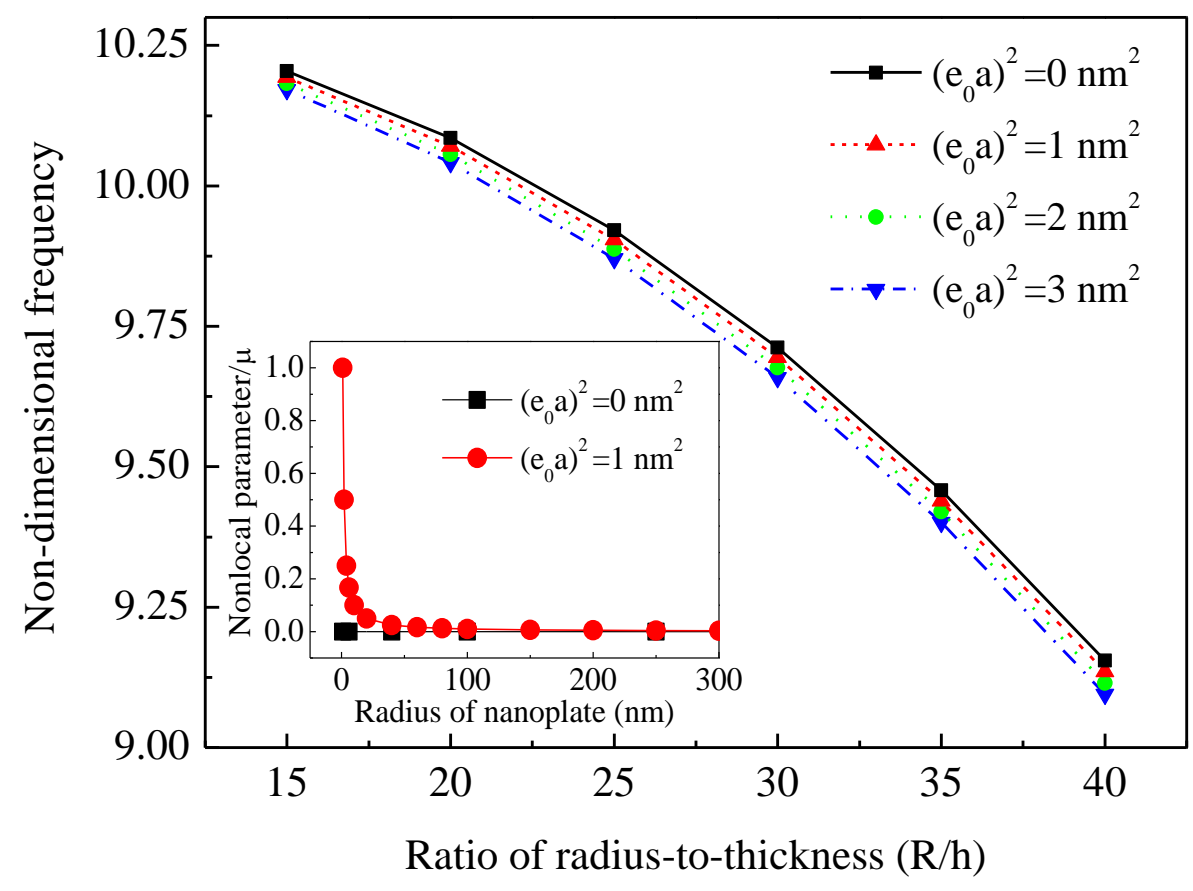

Fig. 10. The effect of nonlocal parameter on non-dimensional frequency for different ratios of radius-to-thickness $\left(K_{2}=0, E^{s}=\tau^{s}=1.0 \mathrm{nN} / \mathrm{nm}, N_{m e}=1 \mathrm{nN} / \mathrm{nm}\right.$,

$$
\left.\Delta T=100 \mathrm{~K}, K_{4}=1, A=0.2\right) .
$$

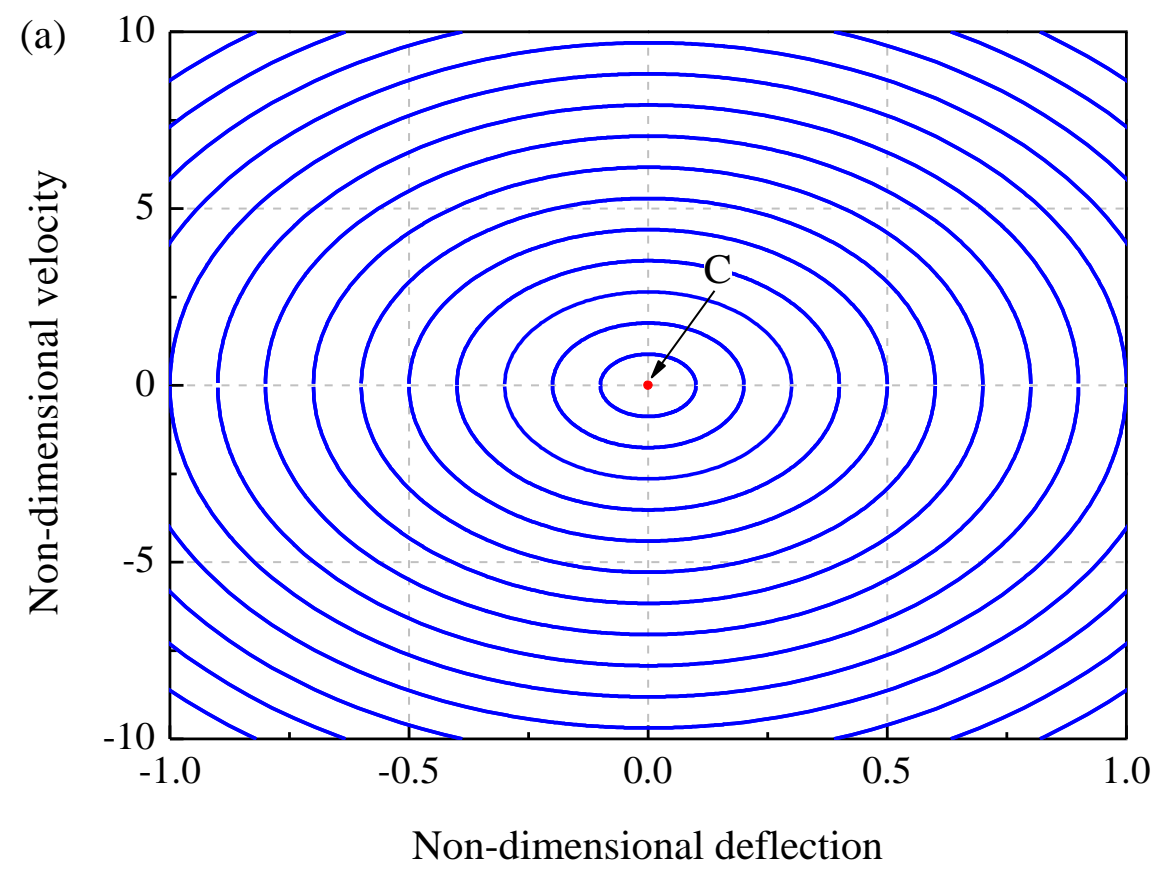



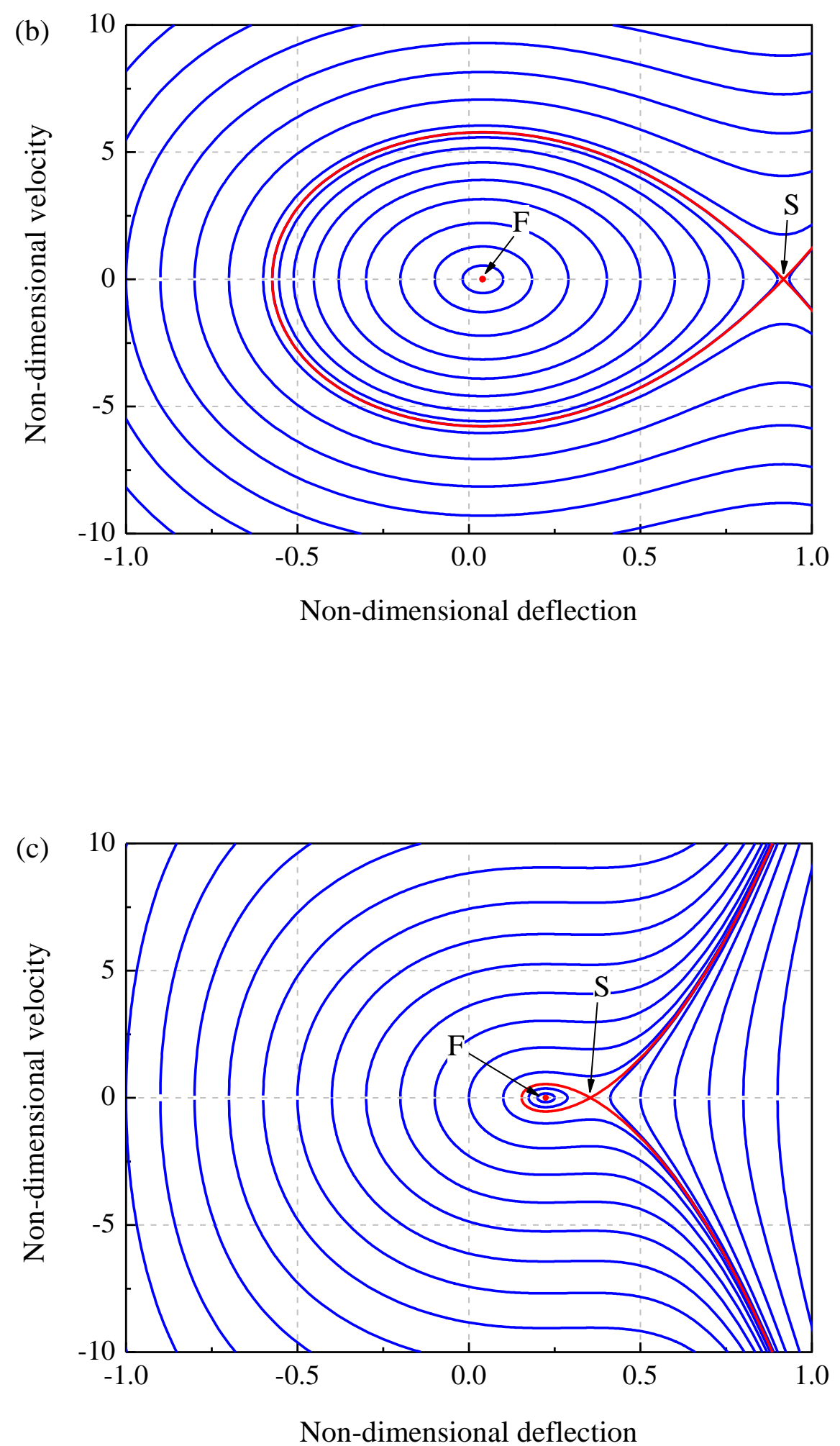


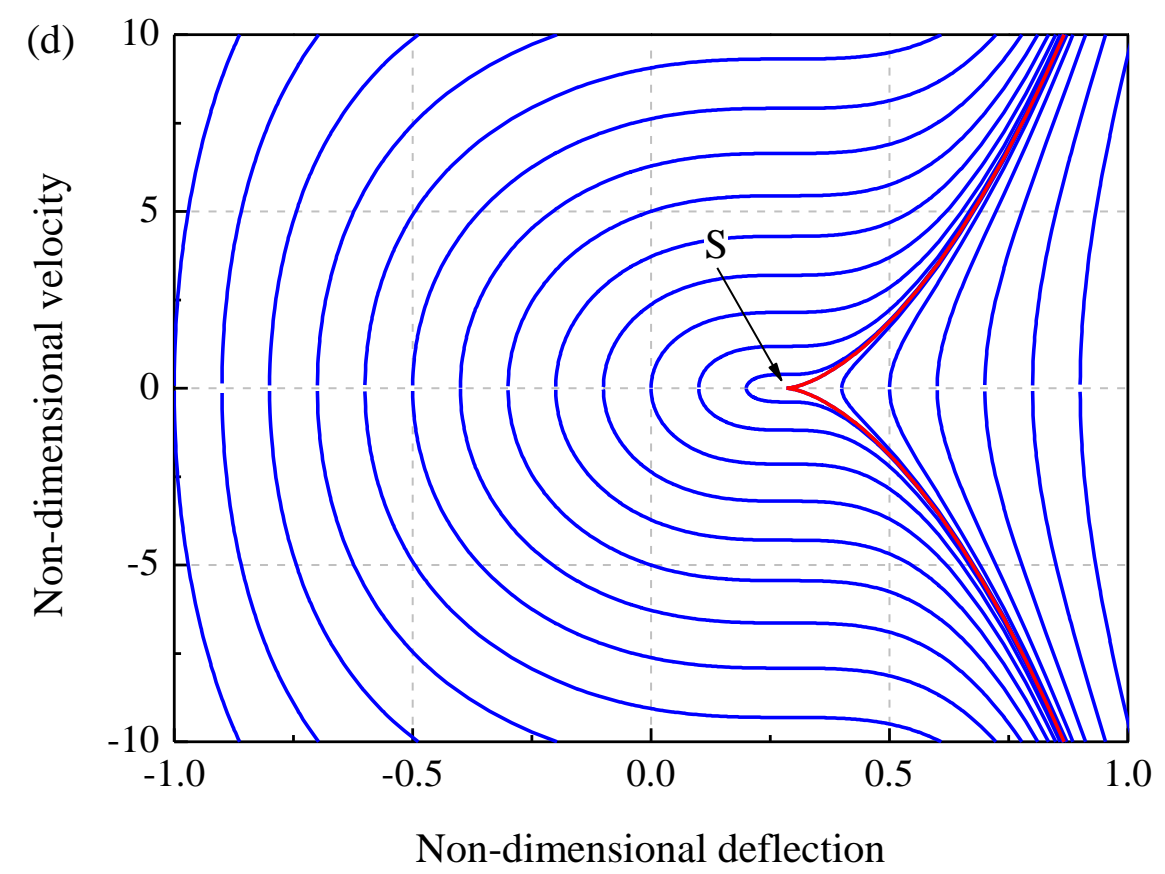

Fig. 11. The transition history of equilibrium points in phase diagram of circular nanoplate when (a) without applied voltage $\left(K_{2}=0\right)$ and Casimir force, (b) small voltage $\left(K_{2}=3.0\right)$, (c) near pull-in voltage $\left(K_{2}=17.8\right)$, and (d) at the pull-in state $\left(K_{2}=19.23\right)$. 


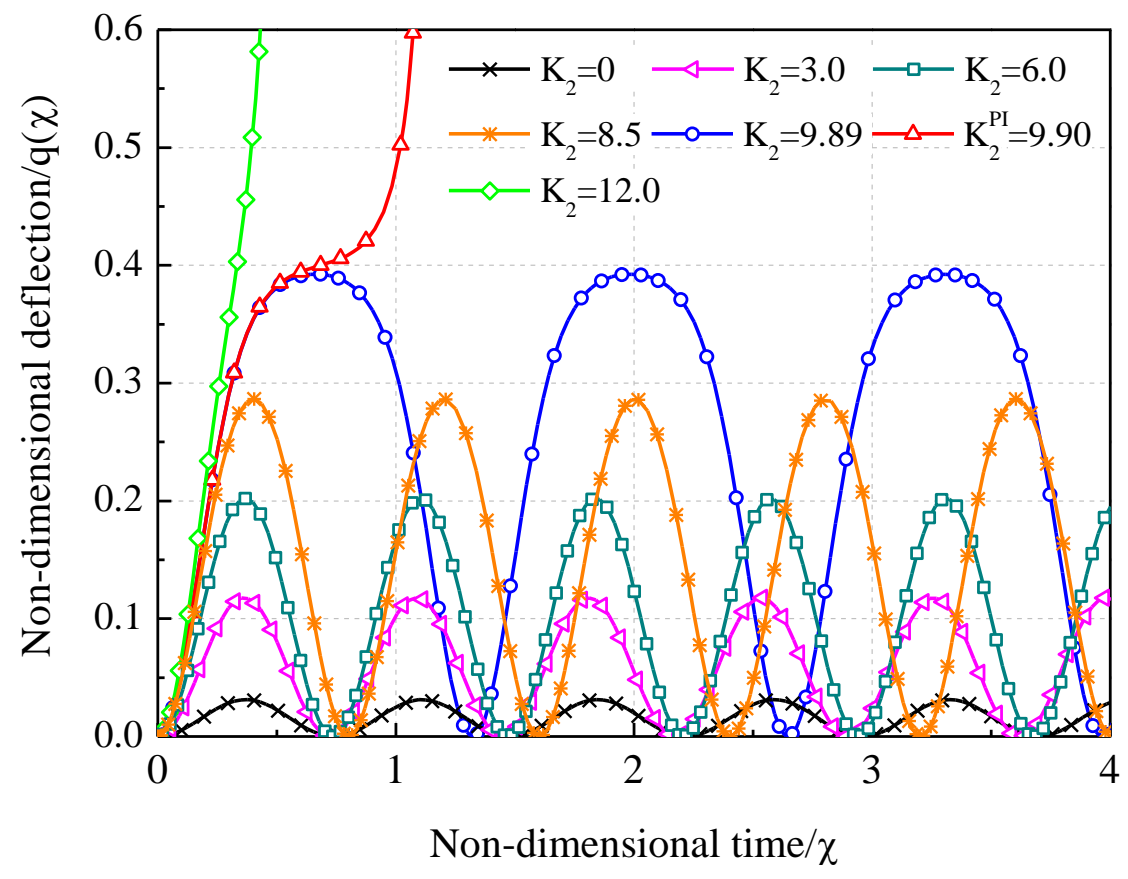

Fig. 12. The time history responses of circular nanoplate under applied voltage and Casimir force $\left(E^{s}=10.605 \mathrm{nN} / \mathrm{nm}, \tau^{s}=1.0 \mathrm{nN} / \mathrm{nm}, \Delta T=100 \mathrm{~K}, N_{m e}=1 \mathrm{nN} / \mathrm{nm},\left(e_{0} a\right)^{2}=1 \mathrm{~nm}^{2}, K_{4}=1\right)$.

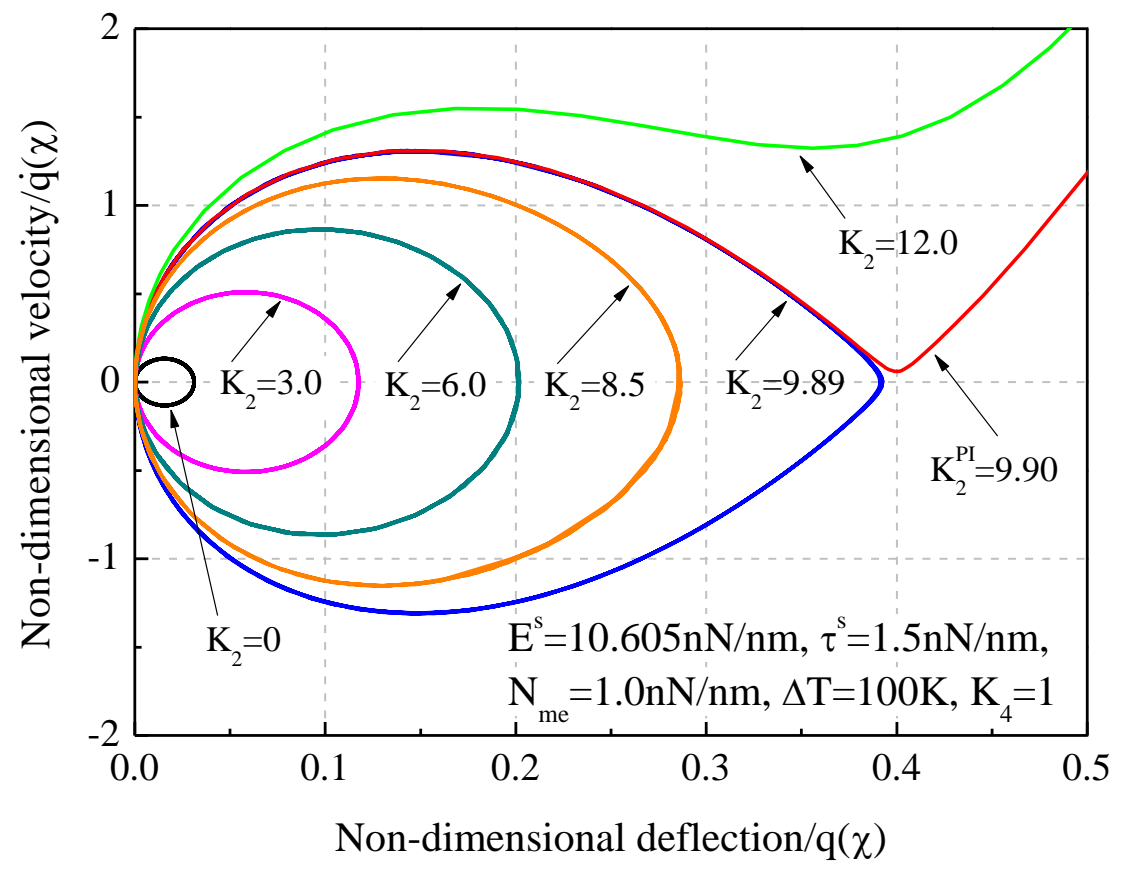

Fig. 13. The phase diagram of circular nanoplate under applied voltage and Casimir force $\left(E^{s}=10.605 \mathrm{nN} / \mathrm{nm}, \tau^{s}=1.0 \mathrm{nN} / \mathrm{nm}, \Delta T=100 \mathrm{~K}, N_{m e}=1 \mathrm{nN} / \mathrm{nm},\left(e_{0} a\right)^{2}=1 \mathrm{~nm}^{2}, K_{4}=1\right)$. 


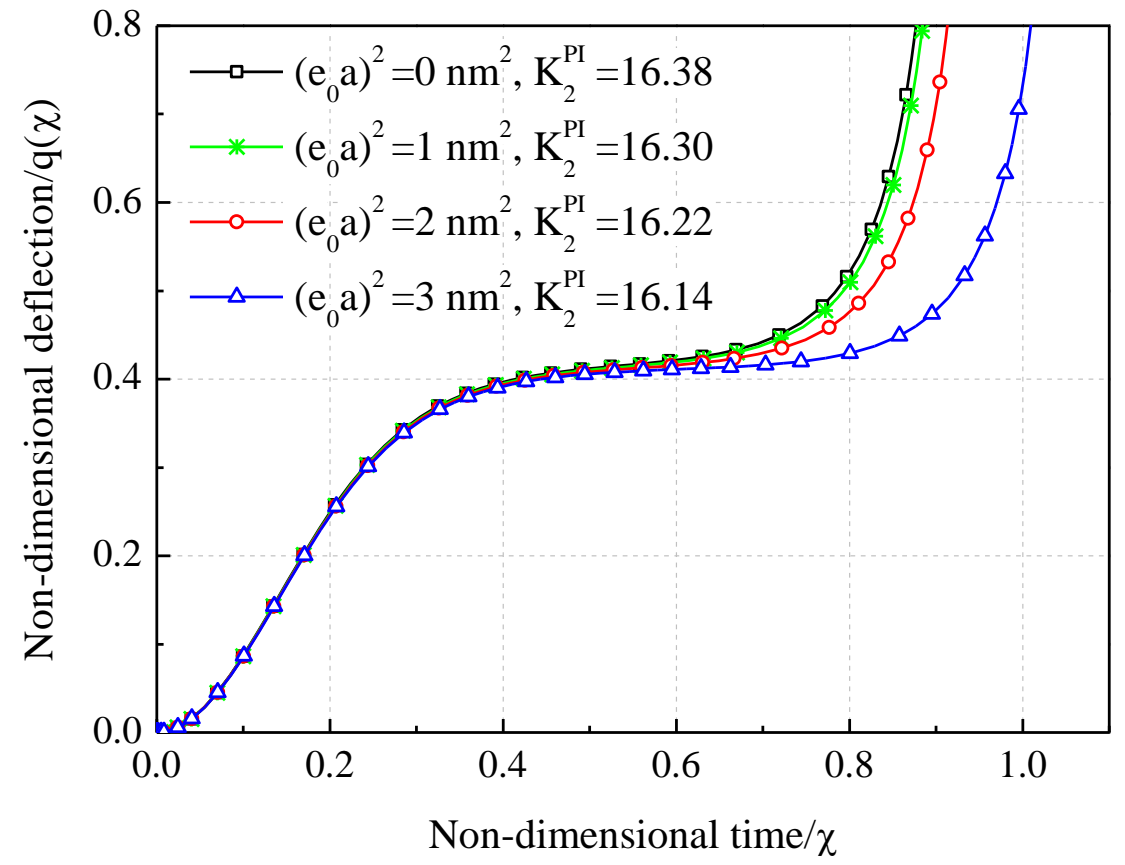

Fig. 14. The small scale effect on time history response of circular nanoplate at the dynamic pull-in state $\left(E^{s}=\tau^{s}=0, N_{m e}=\Delta T=0, K_{4}=1\right)$.

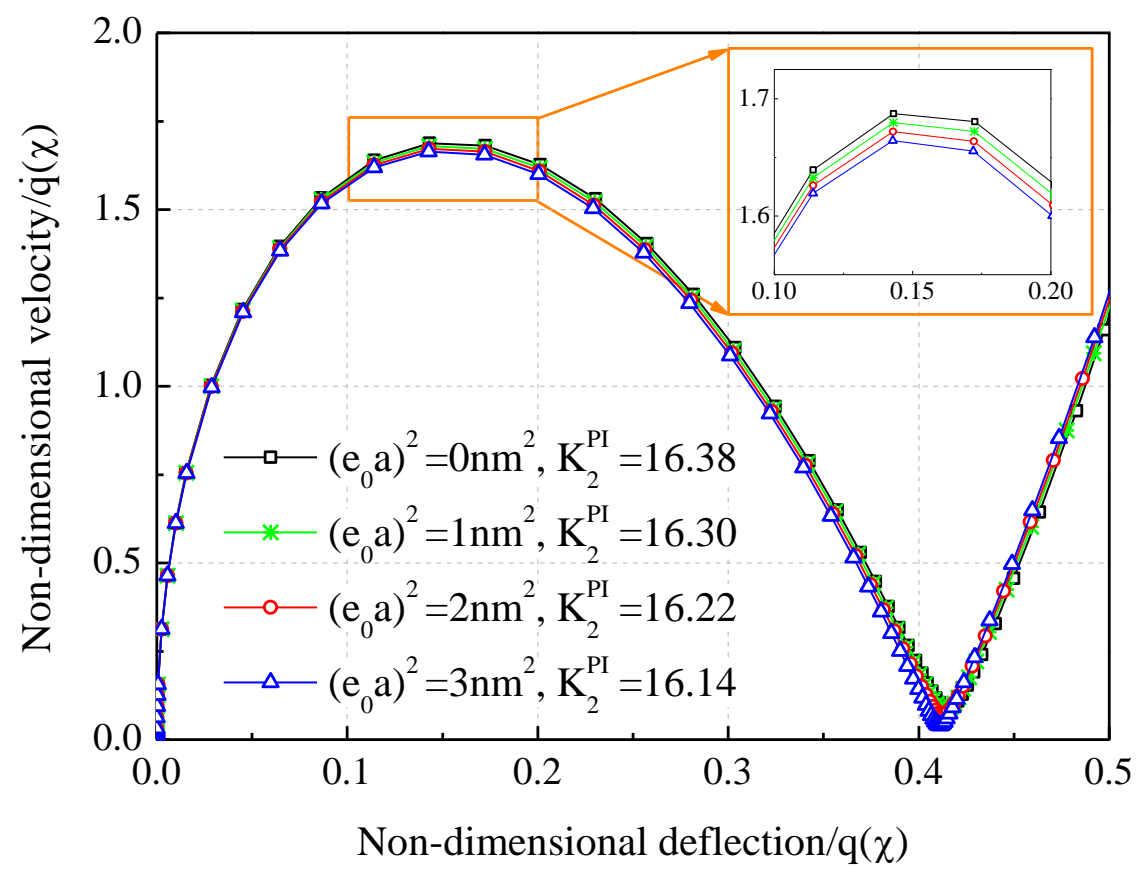

Fig. 15. The small scale effect on the instable phase diagram of circular nanoplate at the dynamic pull-in state $\left(E^{s}=\tau^{s}=0, N_{m e}=\Delta T=0, K_{4}=1\right)$. 
Table 1. Influence of Casimir force on deflection by HPM and numerical method.

\begin{tabular}{|c|c|c|c|c|c|}
\hline \multirow{2}{*}{$\begin{array}{l}\text { Initial } \\
\text { amplitude: } \\
\text { A }\end{array}$} & \multirow{2}{*}{$\begin{array}{l}\text { Electrostatic } \\
\text { voltage: } \\
K_{2}\end{array}$} & \multicolumn{2}{|c|}{ without Casimir force $\left(\alpha_{4}=0\right)$} & \multicolumn{2}{|c|}{ with Casimir force $\left(\alpha_{4}=1\right)$} \\
\hline & & $2^{\text {nd }} H P M$ & Numerical & $2^{\text {nd }} H P M$ & Numerical \\
\hline \multirow{2}{*}{0} & 2.5 & 0.0129089 & 0.0129039 & 0.0180201 & 0.0179980 \\
\hline & 5.0 & 0.0257259 & 0.0256876 & 0.0308019 & 0.0307126 \\
\hline \multirow{2}{*}{0.05} & 2.5 & 0.0120472 & 0.0119438 & 0.0061568 & 0.0058273 \\
\hline & 5.0 & 0.0020747 & 0.0020638 & 0.0081619 & 0.0080864 \\
\hline \multirow{2}{*}{0.10} & 2.5 & 0.0368892 & 0.0366446 & 0.0301626 & 0.0293288 \\
\hline & 5.0 & 0.022177 & 0.0212676 & 0.0149420 & 0.0140721 \\
\hline \multirow{2}{*}{0.15} & 2.5 & 0.0616543 & 0.0602191 & 0.0541304 & 0.0525407 \\
\hline & 5.0 & 0.0459902 & 0.0443494 & 0.0393859 & 0.0385215 \\
\hline \multirow{2}{*}{0.20} & 2.5 & 0.0863587 & 0.0856631 & 0.0768227 & 0.0754041 \\
\hline & 5.0 & 0.0698275 & 0.0671754 & 0.0609557 & 0.0582059 \\
\hline
\end{tabular}

Table 2. Geometrical and material parameters of micro-plate for validation.

\begin{tabular}{lll}
\hline Parameters & Symbol & Value/unit \\
\hline Radius & $R$ & $250 \mu \mathrm{m}$ \\
Thickness & $h$ & $20 \mu \mathrm{m}$ \\
Initial gap & $g_{0}$ & $1 \mu \mathrm{m}$ \\
Young's modulus & $E$ & $169 \mathrm{GPa}$ \\
Poisson's ratio & $v$ & 0.30 \\
\hline
\end{tabular}


Table 3. Comparison of dynamic pull-in voltages from various methods.

\begin{tabular}{lllll}
\hline Method & Step size $(\mathrm{V})$ & $\begin{array}{l}\text { Dynamic pull-in } \\
\text { voltage }(\mathrm{V})\end{array}$ & $\Delta(\%)$ & Reference \\
\hline $\begin{array}{l}\text { Experiment } \\
\begin{array}{l}\text { Finite element } \\
\text { method (FEM) }\end{array}\end{array}$ & 311.6 & $\begin{array}{l}\text { Osterberg } \\
{[61]}\end{array}$ \\
& $\delta \bar{V}_{e}=0.1$ & 315.1 & $\begin{array}{l}\text { Raback and Pursula } \\
{[62]}\end{array}$ \\
$\begin{array}{l}\text { Step-by-step } \\
\text { linearization } \\
\text { method (SSLM) } \\
\text { (N=20) }\end{array}$ & $\delta \bar{V}_{e}=0.05$ & 315.00 & 1.12 & \\
& $\delta \bar{V}_{e}=0.01$ & 314.89 & 1.09 & Rashvand et al. \\
& $\delta \bar{V}_{e}=0.001$ & 314.860 & 1.06 & \\
$\begin{array}{l}\text { Homotopy } \\
\text { perturbation }\end{array}$ & & 1.05 & \\
method (HPM) & 314.878 & 1.05 & Present work \\
\hline
\end{tabular}

$$
\text { -With: } \Delta(\%)=\frac{\mathrm{ABS}(\text { Experiment }-\mathrm{Num})}{\text { Experiment }} \times 100
$$

Table 4. The material and geometrical parameters of circular nanoplate.

\begin{tabular}{lll}
\hline Parameters & Symbol & Value/unit \\
\hline Thickness & $h$ & $5 \mathrm{~nm}$ \\
Radius & $R$ & $150 \mathrm{~nm}$ \\
Initial gap & $g_{0}$ & $6 \mathrm{~nm}$ \\
Young's modulus & $E$ & $210 \mathrm{GPa}$ \\
Poisson's ratio & $v$ & 0.27 \\
Mass density & $\rho$ & $2370 \mathrm{~kg} / \mathrm{m}^{3}$ \\
CTE & $\alpha$ & $2.3 \times 10^{-6} \mathrm{~K}^{-1}$ \\
Permittivity of vacuum & $\varepsilon_{0}$ & $8.854 \times 10^{-12} \mathrm{~F} / \mathrm{m}$ \\
\hline
\end{tabular}




\section{Graphical abstract}

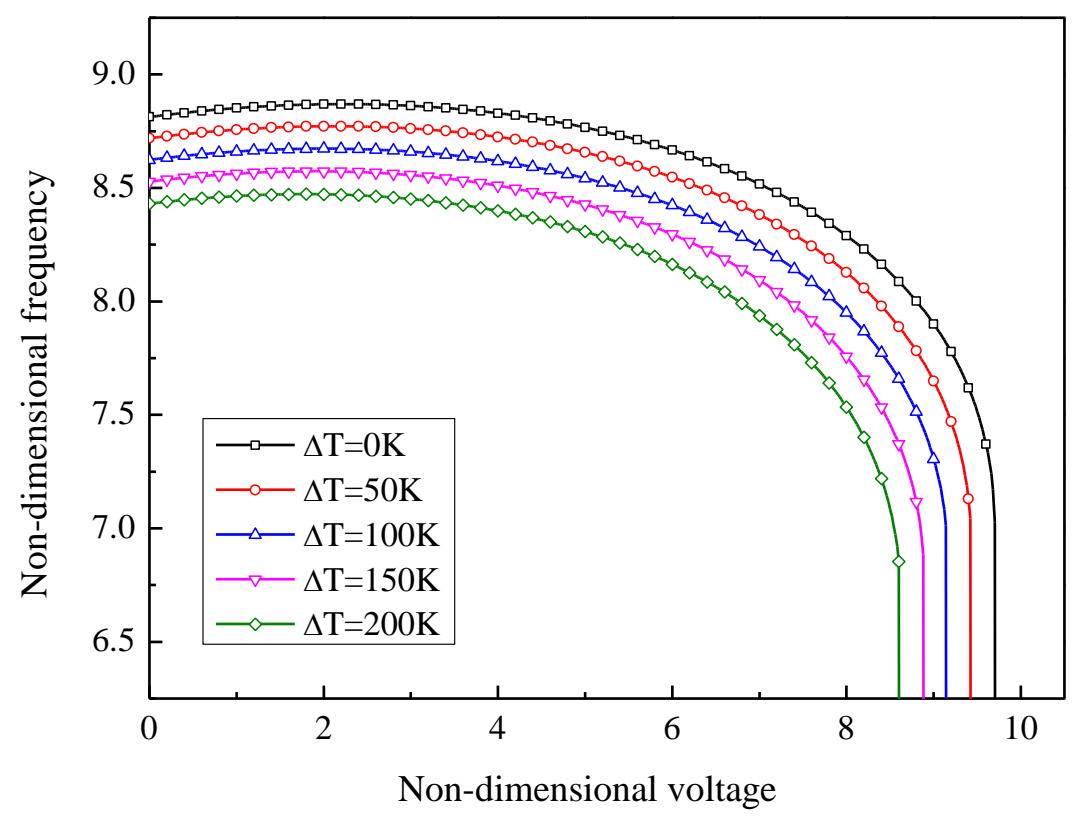

Fig.1. The influences of temperature change on curves of non-dimensional frequency and voltage

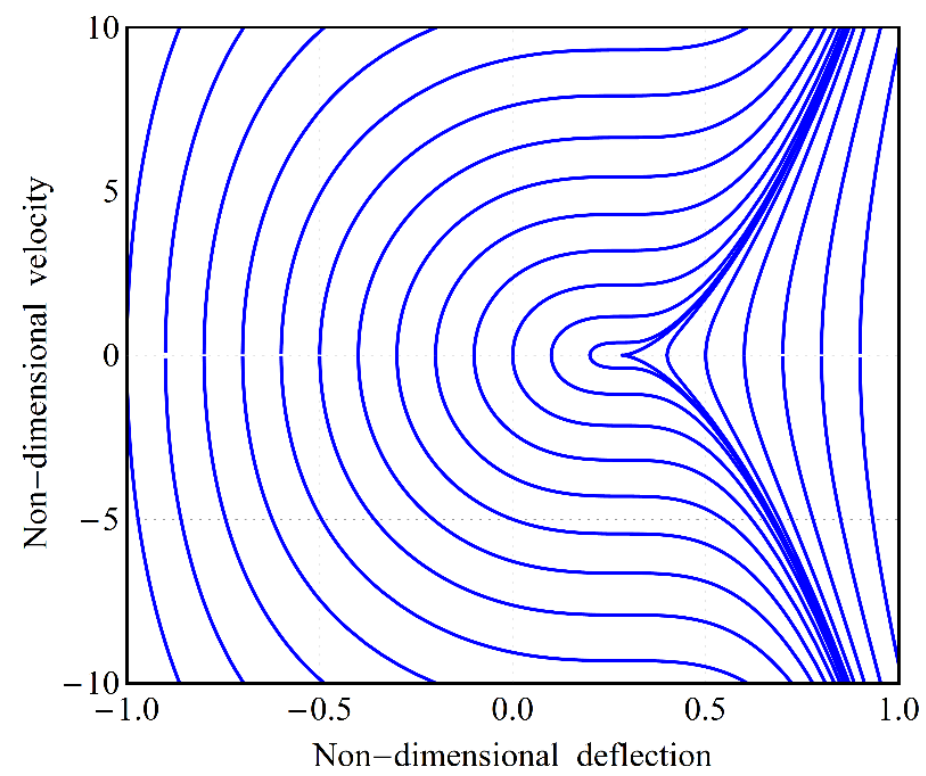

Fig. 2. The phase diagram of circular nanoplate with applied voltage at the pull-in voltage $\left(K_{2}=19.23\right)$. 